\title{
Article \\ An Explainable DL-Based Condition Monitoring Framework for Water-Emulsified Diesel CR Systems
}

\author{
Ugochukwu Ejike Akpudo (1) and Jang-Wook Hur *
}

check for updates

Citation: Akpudo, U.E.; Hur, J.-W. An Explainable DL-Based Condition Monitoring Framework for Water-Emulsified Diesel CR Systems. Electronics 2021, 10, 2522. https:// doi.org/10.3390/electronics10202522

Academic Editors: Paolo Castaldi and Silvio Simani

Received: 23 August 2021

Accepted: 12 October 2021

Published: 15 October 2021

Publisher's Note: MDPI stays neutral with regard to jurisdictional claims in published maps and institutional affiliations.

Copyright: (c) 2021 by the authors. Licensee MDPI, Basel, Switzerland. This article is an open access article distributed under the terms and conditions of the Creative Commons Attribution (CC BY) license (https:// creativecommons.org/licenses/by/ $4.0 /)$.
Department of Mechanical Engineering (Department of Aeronautics, Mechanical and Electronic Convergence Engineering), Kumoh National Institute of Technology, 61 Daehak-ro (yangho-dong), Gumi 39177, Korea; akpudougo@gmail.com

* Correspondence: hhjw88@kumoh.ac.kr

\begin{abstract}
Despite global patronage, diesel engines still contribute significantly to urban air pollution, and with the ongoing campaign for green automobiles, there is an increasing demand for controlling/monitoring the pollution severity of diesel engines especially in heavy-duty industries. Emulsified diesel fuels provide a readily available solution to engine pollution; however, the inherent reduction in engine power, component corrosion, and/or damage poses a major concern for global adoption. Notwithstanding, on-going investigations suggest the need for reliable condition monitoring frameworks to accurately monitor/control the water-diesel emulsion compositions for inevitable cases. This study proposes the use of common rail (CR) pressure differentials and a deep one-dimensional convolutional neural network (1D-CNN) with the local interpretable modelagnostic explanations (LIME) for empirical diagnostic evaluations (and validations) using a KIA Sorento 2004 four-cylinder line engine as a case study. CR pressure signals were digitally extracted at various water-in-diesel emulsion compositions at various engine RPMs, pre-processed, and used for necessary transient and spectral analysis, and empirical validations. Results reveal high model trustworthiness with an average validation accuracy of $95.9 \%$.
\end{abstract}

Keywords: common rail; fault detection and isolation; water-emulsified diesel fuel; condition monitoring; convolutional neural network

\section{Introduction}

For several decades, diesel engines have served profitably for high-power energy/power generation across industries, and with several innovations, their cost efficiency, output optimization, and process design/control have significantly improved [1-3]. On the downside, the emissions from these engines contain high amounts of harmful compoundsnitrogen oxides (NOx), particulate matter (PM), and carbon monoxide (CO). These pollutants contribute nearly $30 \%$ of greenhouse effects and several health and environmental problems. Although an aggressive campaign is ongoing for renewable non-combustible energy sources; particularly for transportation, the necessary resources required for such a transition are yet to be fully harnessed on a global scale while the increasing energy demand still remain [2,4]. Studies on the Asia-Pacific and European regions suggest there will be a $75 \%$ increase in energy demand for transportation by 2040 . This also suggests there would be an $85 \%$ increase in demand for heavy-duty diesel fuels and a $10 \%$ decrease in demand for gasoline fuels [5].

The impact of fuel quality on combustion engine efficiency, power, and durability cannot be overemphasized; however, there is a need to minimize the amount of pollution while maintaining engine efficiency [6,7]. A common, cost-efficient, and reliable emission control method for diesel engines is the use of water-in-diesel emulsified fuels. This is because the physio-chemical properties of emulsion fuels disfavours NOx, CO, and PM productionthe major pollution-causing compounds. Nonetheless, the composition percentage of water by volume of diesel should be kept in check to avoid engine power losses, component 
corrosion, seal damage, and other injection system problems [8-10]. Consequently, there is a need for accurate condition monitoring methods for emulsified diesel fuel engines. Although regular engine checkups and routine maintenance provide long-lasting engine durability, such mechanical methods may be cost-inefficient and unreliable considering the emergence and availability of real-time intelligent alternative methods-data-driven predictive maintenance [11].

Several studies provide some validation on the efficiencies associated with emulsified diesel fuels; yet, only a few works have been recorded on the reliability of using emulsified diesel fuels. Uncertainty and stability issues with mixing conditions (temperature, compositions, etc.) are enough motivation for designing accurate monitoring/diagnostic frameworks to ensure that acceptable hydrophilic-lipophilic balance (HLB) in the emulsion and engine efficiencies are maintained at all times $[12,13]$. To achieve these, studies have shown that leveraging the dynamics and vulnerabilities of CR systems are quite reliable, and with recent advancements in dynamic modelling (which are artificial intelligence (AI)-driven), accurate real-time monitoring and diagnostics can be achieved with minimal false alarm rates $[1,6]$. Beyond the limitations of traditional machine learning techniques like the support vector machines (SVM), random forests and decision trees, DL techniques like the convolutional neural networks (CNN), recurrent neural networks (RNN), etc. come with diverse advantages including big data compatibility, automated feature engineering, and ease-of-use $[14,15]$. On the other hand, processing these signals using befitting signal processing techniques further enhances the accuracy, reliability, and acceptability of the inherent model(s) for practical use $[1,6,15]$.

In our quest for investigating diesel engine behaviour by leveraging the characteristics of CR fluctuations in a water-emulsified fuel diesel engine, our study makes the following contributions:

- An investigative study was conducted on a commercial diesel engine operating on varying water-in-diesel compositions and engine speeds. Using the $C R$ pressure signals, empirical analyses were conducted to understand the characteristics of CR fluctuations over these conditions.

- A hybrid diagnostic scheme is proposed for condition monitoring. This model leverages the discriminative properties of the first-order differential of $\mathrm{CR}$ pressure signals on one hand, and the superior diagnostic property of the 1D-CNN on the other hand.

- Using the LIME algorithm [16], our study provides empirical explanations on the faithfulness of predictions made by the diagnostic model. These explanations/interpretations by the LIME algorithm primarily functions by approximating local predictions with an interpretable model.

To provide a clear road-map to the rest of this paper, the next section presents a detailed overview of the materials and methods employed herein (an overview of CR systems, test engine and fuels, and the experimental procedure). Section 2 provides the proposed diagnostic system model and key theoretical background while Section 4 presents the empirical assessments and validation from the case study. Section 5 summarizes some related works and open issues while Section 6 concludes the paper.

\section{Materials and Methods}

This section presents an overview of the diesel engine CR system and the specifics of the test engine (and fuels) used in this study.

\subsection{Common Rail Injection System}

Figure 1 presents an illustration of a typical high-pressure (HP) injection system consisting of the major parts-HP pump (HPP), CR system, fuel injector, and the electronic control unit (ECU) [17]. The injection process starts via the yellow lines whereby the low-pressure pump (LPP) delivers fuel from the reservoir through a filter to the HPP. The HPP pumps the fuel at a high pressure (in red) to the CR which distributes the fuel to the injector(s) through the accumulator for spraying in the combustion chambers. 


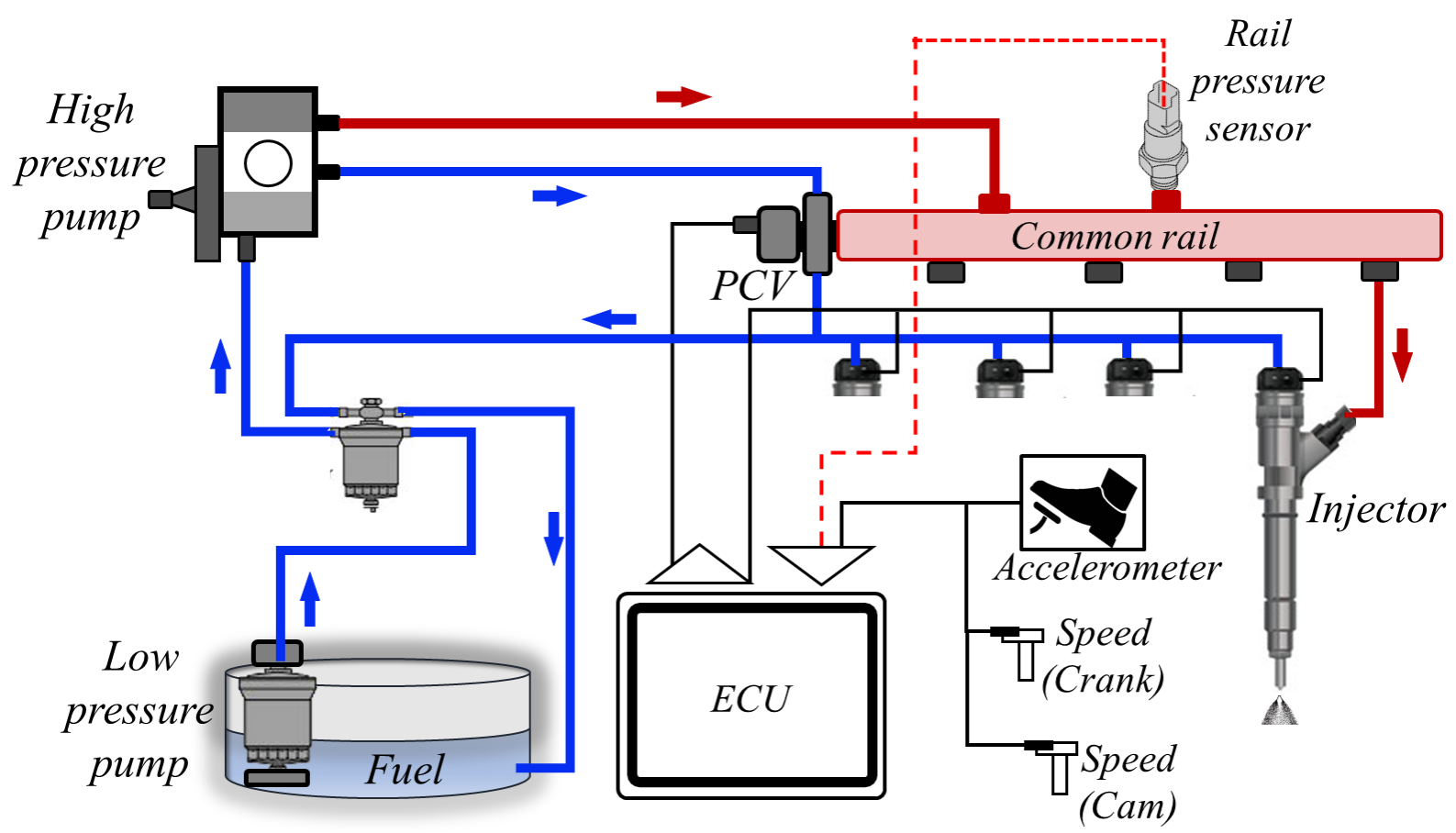

Figure 1. A typical Common rail diesel fuel injection system.

The pressure control valve (PCV) curtails abnormal fuel flow in the CR pipe via a throttling behaviour induced by the ECU. The ECU is primarily the brain of the CR system since it controls the opening and closing of the injectors, and the throttling behaviour of the PCV especially under varying engine speeds. Every spray in the combustion chamber induces an instant pressure drop in the CR pipe which causes the HPP and its regulator to compensate for this pressure drop. These fluctuations in pressure are reflected by the CR sensor and provide reliable condition monitoring measurements.

The CR system dynamics of a diesel engine is quite reflective of the engine's performance and running conditions. The spectral and transient information underlying in the CR signal; when properly harnessed, can provide reliable diagnostic/condition monitoring parameters.

\subsection{Test Engine and Fuel Preparation}

A passenger car, KIA Sorento 2004 model, was used for the proposed study. Figure 2 shows the actual experimental setup while Table 1 provides the engine's specification. The experiment was performed during early winter season at an approximate room temperature of $10^{\circ} \mathrm{C}$ with different emulsion compositions (under varying engine speeds).

Table 1. Test engine specifications.

\begin{tabular}{|c|c|c|c|c|c|c|c|}
\hline Car Model & Engine Type & Bore $\times$ Stroke $(\mathrm{mm})$ & Maximum Power & Maximum Torque (Nm/RPM) & Compression Ratio & Fuel Injection & Aspiration \\
\hline $\begin{array}{l}\text { KIA Sorento } \\
2004\end{array}$ & $\begin{array}{l}\text { In-line, } \\
\text { Four (4) }\end{array}$ & $91 \times 96$ & $\begin{array}{c}138 \text { hp @ } \\
3800 \text { RPM }\end{array}$ & $\begin{array}{l}343 \text { Nm @ } \\
1900 \text { RPM }\end{array}$ & 17.6 & Common Rail & $\begin{array}{l}\text { Turbocharged, } \\
\text { inter-cooled }\end{array}$ \\
\hline
\end{tabular}


(a)

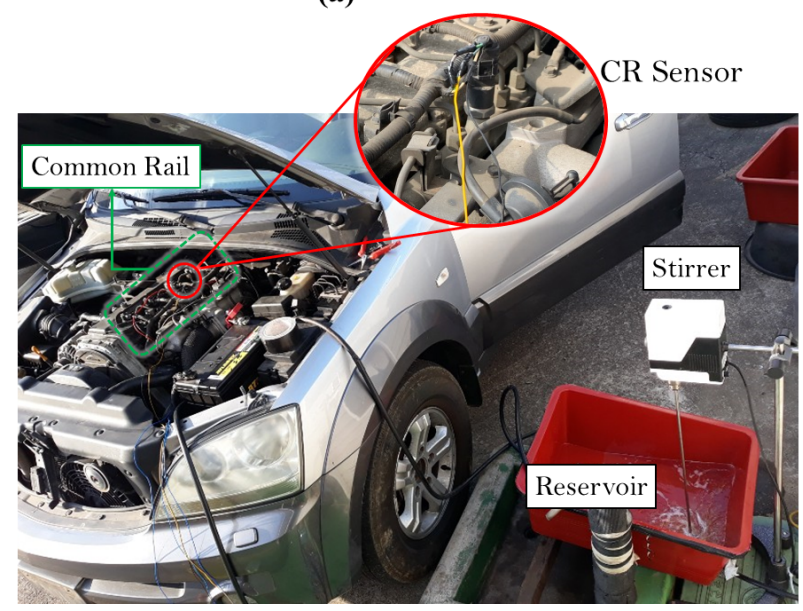

(b)

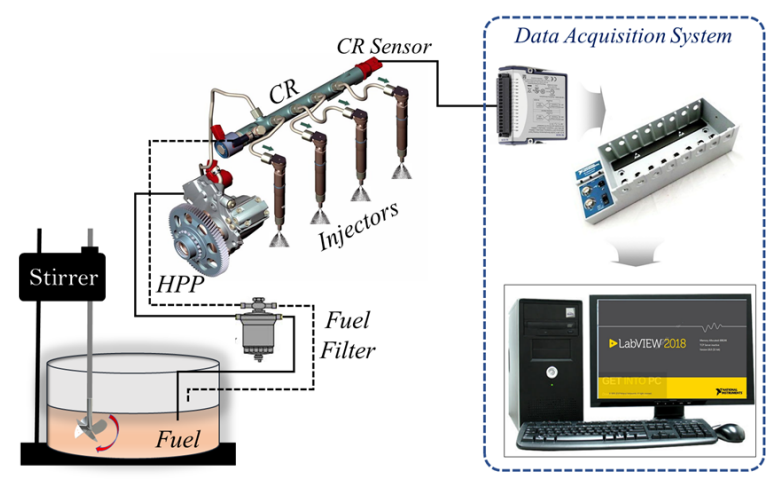

Figure 2. A view of (a) the actual experiment setup, (b) schematic view of the experimental setup.

From the car engine's rail pressure sensor (RPS), pressure measurements were digitally collected via an NI 9228 at $200 \mathrm{~Hz}$ on a NI Compact DAQ 9178 data acquisition system and a LabView environment. The RPS outputs a maximum of $(5 \pm 0.25) \mathrm{V}$ at 2200 bar with a response time $\left(\tau_{10 / 90}\right) \leq 5 \mathrm{mSecs}$ and a maximum overpressure of 1800 bar (rupture pressure $=3000$ bar). Furthermore, the real-time engine temperature data was collected via NI 9214 module at $1 \mathrm{KHz}$ with an RTD thermocouple affixed to the exhaust manifold.

Different water compositions are mixed with diesel to form different emulsion compositions represented as EM- $x \%$ (where $x$ represents the concentration of water by volume in $(100-x) \%$ of diesel. First, the car's fuel tank was isolated and an external custom reservoir was designed for the experiment (see Figure $2 b$ ). To avoid phase separation, the water-emulsified fuels were constantly stirred by an overhead stirrer (OSA-10 made by LK LABKOREA) and tested for stability with a centrifuge. Table 2 shows the different viscosity values of the emulsion compositions at $10^{\circ} \mathrm{C}$.

Table 2. The viscosity of different water-emulsion compositions.

\begin{tabular}{cccccccc}
\hline Emulsion Composition & EM-0\% & EM-1.3\% & EM-1.5\% & EM-2.0\% & EM-5.0\% & EM-10.0\% & EM-20.0\% \\
\hline Kinematic Viscosity (cSt) at $10{ }^{\circ} \mathrm{C}$ & 6.24 & 9.478 & 9.49 & 9.52 & 9.71 & 10.01 & 10.61 \\
\hline
\end{tabular}

As observed, the emulsion viscosity increases linearly as the water composition is increased. This suggests a possible positive correlation between increasing emulsion composition and increased engine stress during combustion. For the respective emulsion conditions, the engine was run for half an hour to ensure the particular emulsion fuel is evenly running through the whole injection system while maintaining a stable temperature. To collect data for the next emulsion composition, a one-hour flush is conducted using the emulsion composition of interest.

\section{Proposed System Model}

Ideally, CR behaviour is highly correlated with the periodic injection process in the combustion chamber; therefore, extracting the CR signals' time-dependent derivatives helps in stationarizing the signals, identifying injection duration, and providing more reliable characteristic parameters for condition monitoring/diagnosis [1]. 


\subsection{CR Signal Pre-Processing}

Figure 3 presents a visual illustration of the proposed DL-based condition monitoring framework. Given the raw CR signals $a(t)=\left\{a(t)_{1}, a(t)_{2}, \ldots, a(t)_{N}\right\}$, the model initializes by standardizing the raw $\mathrm{CR}$ signals using Equation (1):

$$
x(t)_{i}=\frac{a(t)_{i}-\mu}{\sigma}
$$

where $\mu$ is the mean of the distribution while $\sigma$ is the standard deviation of $a(t)$.

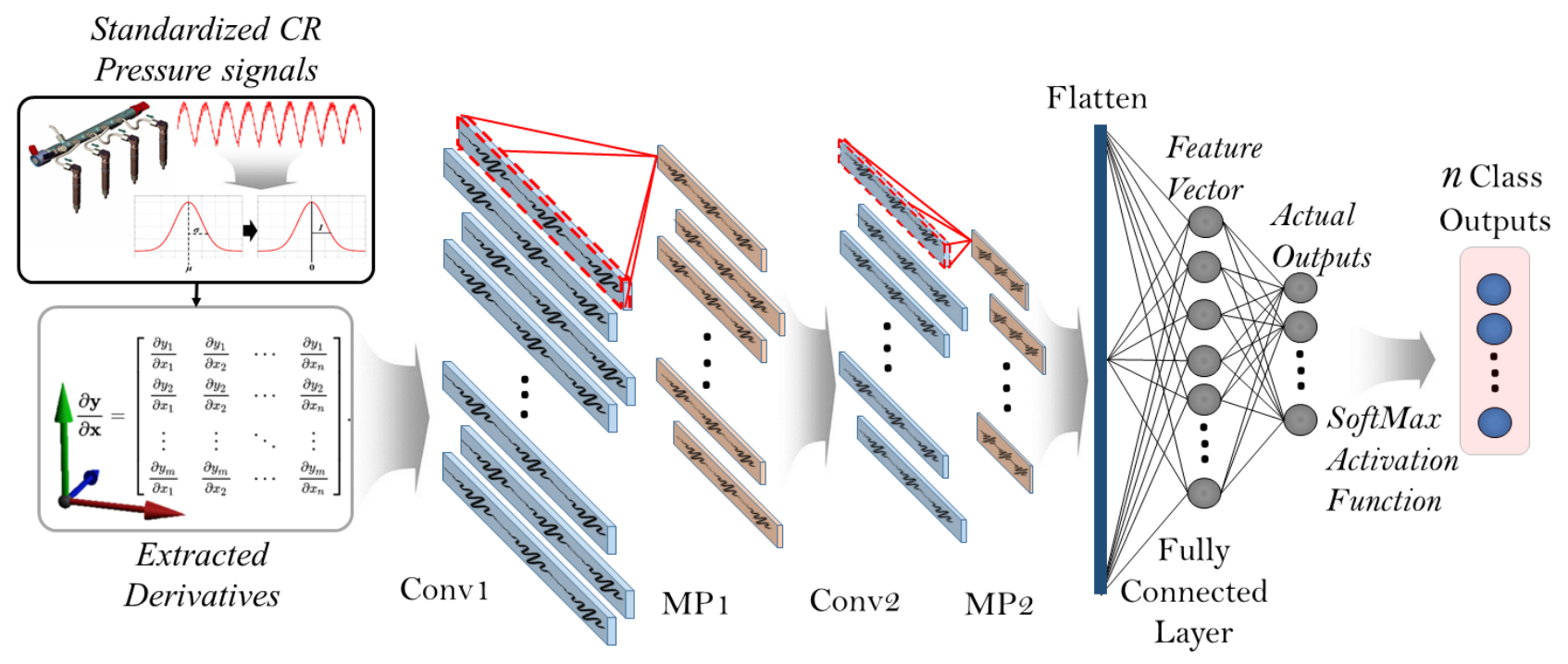

Figure 3. Proposed 1D-CNN-based condition monitoring model.

Next, the model computes the first-order derivatives of standardized signals using Equation (2)

$$
x^{\prime}(t)_{i}=\frac{x\left(t_{i+1}\right)-x\left(t_{i-1}\right)}{2 * \Delta t}
$$

This is an important step for extracting the time-dependent information peculiar to each signal while also eliminating bias, improving stationarity in the signals, and chiefly, improving discriminance in the inputs.

\subsection{D-CNN Diagnostic Model Overview}

Amongst the many ML/DL algorithms, CNNs are unique for their working principlemimicking the human visual cortex for discriminative feature extraction and identification of labels (objects, conditions, etc.). A typical CNN differs from conventional ML algorithms in the context of feature extraction, whereby it extracts features globally through a number of stacked layers and with fully connected layers, can also act as a classifier. This makes the $\mathrm{CNN}$ an all-in-one diagnostic tool since it consists of a feature extractor and classifier.

The uniqueness (and superiority) of CNNs for supervised discriminative predictions have made them very popular for many diagnostic/detection cases including, but not limited to Network Intrusion Detection [18], Theft Detection in Smart Grid Systems [19], speech separation [20], etc. This superiority is attributed to the convolving (Conv) and maxpooling (MP) layers which function as filters for high-level (automatic) feature extraction; and with the fully connected layer(s)—classical artificial neural network (ANN), multi-label predictions can be made [21,22].

As shown in Figure 3, the proposed model's efficiency relies significantly on the classification/diagnostics module which comprises of two Conv and MP layers with rectified linear units (ReLU) in between them- [Conv1-MP1] and [Conv2-MP2], and two fully connected layers with SoftMax-activated outputs for each of the operating conditions. 
Respectively, the Conv layers had 32 and 64 filters with a kernel size of $1 \times 5 \times M$ where $M$ represents the depth of the input signals. the filter size for the $M P$ layers was $1 \times 5$.

Given a set of multi-class $C R$ pressure differentials as inputs$X_{n}^{\prime m}=\left\{\left(x_{1}^{\prime}, y_{1}\right),\left(x_{2}^{\prime}, y_{2}\right), \ldots,\left(x_{n}^{\prime}, y_{n}\right)\right\}$, where $x_{n}^{\prime} \in R^{m}$ and $y_{n} \in\{0,1, \ldots, n\}$, the ReLU-activated outputs (features) from the Conv-MP layers are computed using Equation (3):

$$
f\left(x^{\prime}\right)=\max \left(0, x^{\prime}\right)
$$

The SoftMax-activated predictions from the fully connected layers are obtained using Equation (4) whereby the model returns 1 for highly probable correct label predictions and 0 for otherwise predictions.

$$
f\left(x^{\prime}\right)=\operatorname{SoftMax}\left(x^{\prime}\right)=\frac{e^{x_{i}^{\prime}}}{\sum_{j=1}^{n} e^{x_{j}^{\prime}}}
$$

\subsection{LIME and Performance Evaluation}

Artificial intelligence (AI) has become predominantly preferable across most disciplines and this has motivated the significantly high disfavour on the traditional statistical model-based techniques. CNNs and other DL methods are quite popular for high detection accuracy, ease-of-use, and real-time compatibility; however, issues of interpretability, high dependence on excessive parameters, overfitting/underfitting issues, computational cost (and complexity), and the magical defiance from fundamental statistical theory made them practically unreliable for industrial applications [23]. Quite interestingly, the need for interpretability and explainability of these black-box models has motivated the development of explainable paradigms for empirically assessing the predictive trustworthiness of these models.

Although performance metrics like accuracy, F1-score, precision, recall, etc. provide reliable paradigms for assessing the predictive performance of classifiers, relying on these global assessment metrics may result in fatal consequences under real-life situations. The complex neuron-neuron and layer-layer connections deter human understanding of the rationale behind the models predictive accuracy. The question-Why should I trust this model? (even with mouth-watering accuracies) only verify the need for providing an explanation to the complexities found in black-box models. Often times, ANNs learn the complex correlations between inputs and make their judgement based on them; sadly, if humans cannot detect these correlations (and control them) the models would most probably make false predictions in real-world applications. Several methods have been proposed for explaining the logic behind DL model decisions without us knowing how/when/where such wrong predictions were made. These include the assessment of feature importance, layer-wise relevance propagation (LRP), LIME, Shapley additive explanations, and feature visualization [24,25]. Among these, LIME provides a reliable paradigm for locally explaining the logic behind the proposed model's decisions/predictions and was employed in this study.

As proposed by Ribeiro et al. [16], LIME explains a model's predictions in a faithful manner by approximating it locally with an interpretable model. In other words, it explains individual sample predictions by returning coefficients based on weightages that it assigns each of the features. The local interpretability effectiveness of LIME provides a strong avenue for assessing the prediction of each input sample/feature. This in turn, makes it possible to assess each feature's contribution to a prediction for any arbitrary sample. Originally, the effectiveness of LIME was assessed on traditional ML-based algorithms [16]; however, we have expanded its capability for providing explanation for DL-based models with reliable results. 


\section{Results and Empirical Analyses}

For each of the emulsion compositions summarised in Table 2, it was observed that the exhaust temperature increased as the engine speed is increased in the range- $(1200$, 1500, 1700, and 2000) RPMs; however, as Figure 4 shows, a cooling effect is observed from EM-2.0\% (in green) through EM-5.0\% (in blue) and then to EM-10.0\% (in red) while for EM-20.0\% (in black), the engine seemed to be overheating in compensation for the poor combustion process and increased mechanical stress on the engine which collectively, were as a result of the excessive water concentration.

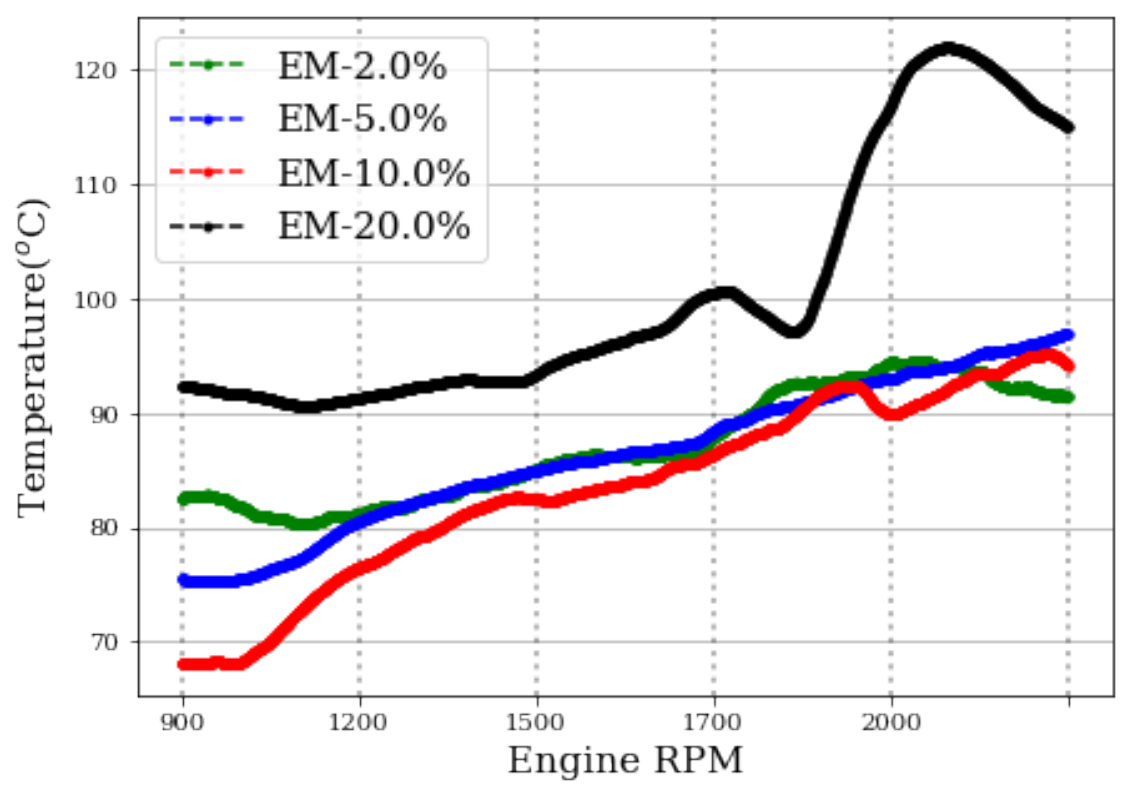

Figure 4. Exhaust temperature for different emulsion compositions at increasing engine speeds.

Unfortunately, the temperature history for EM-0\%, EM-1.3\%, and EM-1.5\% were not collected as a result of sensor line malfunction during the experiment.

\subsection{CR System Characteristics}

To validate the need for extracting the first-order $\mathrm{CR}$ pressure differentials as inputs for the proposed model, this subsection presents a spectral analysis of the raw CR signals and their respective differentials. As a tool for investigation, the fast Fourier transform (FFT) and power spectral density (PSD) are employed for analyzing the spectral behaviour of the CR system. Invariably, this information provide intuitive knowledge on the uniqueness/discriminance, energy distribution, and characteristics of the respective CR signals for condition monitoring, while also creating an avenue for stationarizing the signals-another key importance of extracting the CR pressure differentials.

A detailed report on the $\mathrm{CR}$ signals for different emulsion compositions at different speeds is provided in Figures A1-A5 (Appendix A). It is observed in the figures that for the $\mathrm{CR}$ pressure signals at different emulsion compositions (far left column), different spectra are obtained; revealing the effect the constituent frequencies from the FFT outputs (middle column) and energy densities from the PSD plots (far right column). Although the respective spectra are quite intuitive, the effect of non-stationarity in the signals are observed in the lower frequency regions. This hints on a high correlation/similarity between the different conditions - an undesired characteristic needed for accurate diagnostic/condition monitoring. In contrast, Figures A6-A10 (Appendix B) which summarise the CR pressure differentials for different emulsion compositions at different speeds, provides better spectral representations for the different emulsion conditions. With unique frequency distributions across the emulsion compositions (and speeds), the discriminance needed from the signals for accurate condition monitoring is increased. Furthermore, it is observed on the left 
columns of the Figures A6-A10 (Appendix B) that the differentials are stationary with a zero mean value.

To summarise the spectral behaviour of the CR system at the various emulsion compositions, selected spectral parameters for analysing non-statioanry signals were employed on the raw CR pressure signals-spectral centroid $\left(\mu_{1}\right)$, root mean square frequency (RMSF), spectral skewness (SS), and spectral kurtosis $\left(K_{f}\right)$, and are defined in Equations (5)-(8), respectively.

$$
\begin{gathered}
\mu_{1}=\frac{\sum_{k=b_{1}}^{b_{2}} f_{k} s_{k}}{\sum_{k=b_{1}}^{b_{2}} s_{k}} \\
R M S F=\sqrt{\frac{\sum_{k=b_{1}}^{b_{2}}\left(f_{k}\right)^{2}}{\sum_{k=b_{1}}^{b_{2}} s_{k}^{2}}} \\
\mathrm{SS}=\frac{\sum_{k=b_{1}}^{b_{2}}\left(f_{k}-\mu_{1}\right)^{3} s_{k}}{\left(\mu_{2}\right)^{3} \sum_{k=b_{1}}^{b_{2}} s_{k}} \\
K_{f}=\frac{\left\langle|S(t, f)|^{4}\right\rangle}{\left\langle|S(t, f)|^{2}\right\rangle^{2}}-2, \quad f \neq 0
\end{gathered}
$$

where $f_{k}$ represents the magnitude of bin number $k$, $s_{k}$ represents the center frequency of $k$, $b_{1}, b_{2}$ are the band edges in bins. $\langle|S(t, f)|\rangle$ is the short-time Fourier transform (STFT) of the signals.

These parameters further reflect the CR dynamics at the different emulsion and speed conditions [26]. The spectral centroid measures signal's spectral position and can provide a comparative estimation of the vigorousness in the CR system while RMSF reflects the overall energy level across the spectra. Spectral skewness measures the symmetry of the spectrum around its arithmetic mean while spectral kurtosis reveal how the impulsiveness of the $C R$ pressure signals vary with frequency. Ideally, the skewness return a zero value for a normal distribution and high (positive) values for signals with substantial energy. Figure 5a-d show the comparison using these parameters for different emulsion compositions at increasing engine speeds.

Observing the spectral centroid in Figure 5a suggests that the spectral centroid values for EM-1.3\%-EM-2.0\% seem to be within acceptable ranges for lower engine speeds (900-1500 RPM) regardless of the increasing CR vigorosness caused by W/D emulsions. On the other hand, Figure $5 \mathrm{~b}$ reveals an overall reduction of $\mathrm{CR}$ energy levels are due to increasing W/D emulsions at engine idling condition (900 RPM). There is no significant impact of increasing the engine speed at these conditions on the energy levels, except for high emulsion compositions-EM-10.0\% (and above), where the energy levels decrease at increased engine speeds.

As shown in Figure 5c, the lower spectral kurtosis observed for EM-1.3\%-EM-20.0\% at lower engine speeds (900-1500 RPM) suggest low impulsiveness of the signals unlike for EM- $0 \%$. These are also supported by the signals shapes revealed by the spectral skewness values in Figure $5 \mathrm{~d}$ which provide intuitive information about the contrasting signal waveforms across the different emulsion compositions at different engine speeds. The compared skewness values in Figure 5d further reveals that small emulsion compositions (EM-1.3\%) at slow car speeds (around 1200 RPM of engine speed) produce CR pressure signals similar to using clean diesel fuel (EM-0\%); however,at higher engine RPMs (1500-2000 RPM), the correlation between the signal shapes at increasing water-emulsion composition (and increasing speeds) is unclear. 
(a)

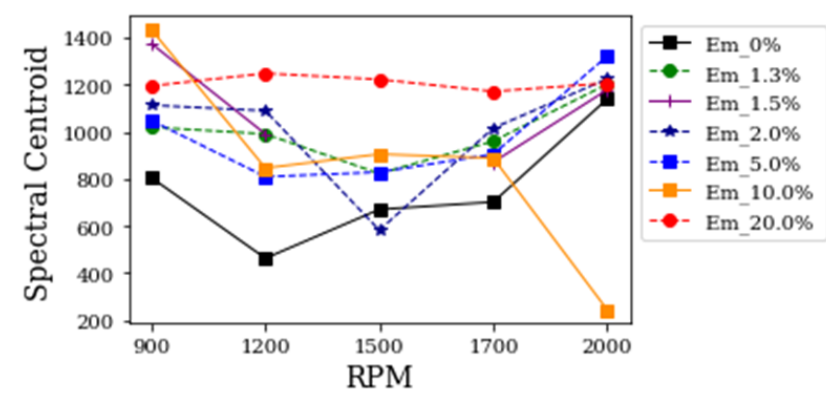

(c)

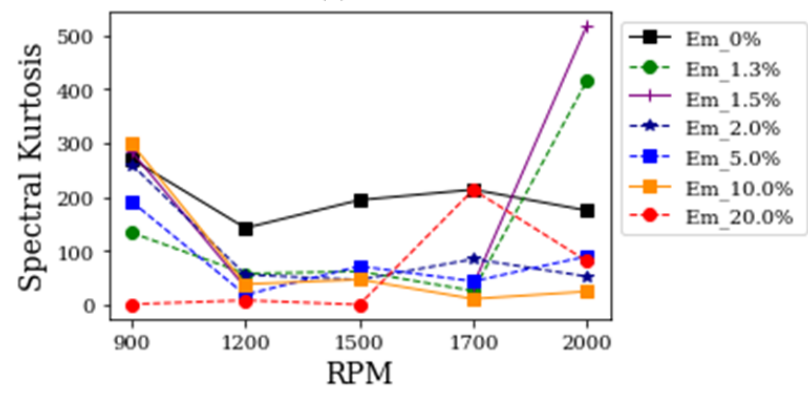

(b)

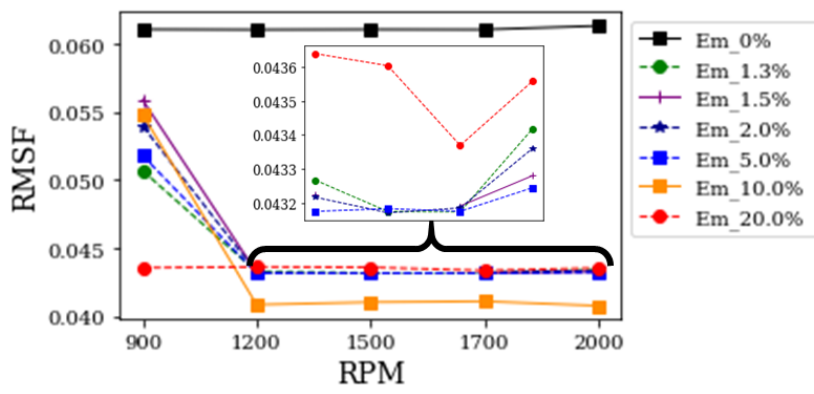

(d)

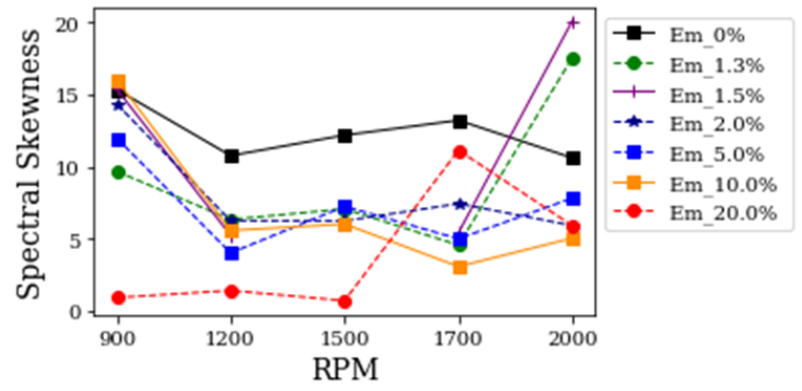

Figure 5. Spectral parameters of CR pressure differentials for different emulsion compositions (a) spectral Centroid, (b) RMSF, (c) Spectral kurtosis, and (d) Spectral skewness.

\subsection{CNN-Based Diagnosis}

The developed model accepts processed CR pressure measurements and outputs normalized class labels. The pressure differentials for each emulsion composition were prepared to form $[m \times d \times n]$ matrices where $m, d, n$ represent the number of training/test examples, number of time periods, and engine speeds. Consequently, for the training and test datasets, $\left[m_{\text {train }} \times 50 \times 5\right]$ and $\left[m_{\text {test }} \times 50 \times 5\right]$ were constructed whereby the $d=50$ samples correspond to 0.1 time periods ( 1 time periods $=5 \mathrm{KHz}$ ).

Using the training set, the developed 1D-CNN model whose architecture is summarised in Section 3.2 was employed with a 0.5 dropout over 50 iterations. The results of the training process is summarised in Figure 6 which shows the validation/test accuracy, loss, recall, precision, and F1-score over the 50 iterations for a single training instance.

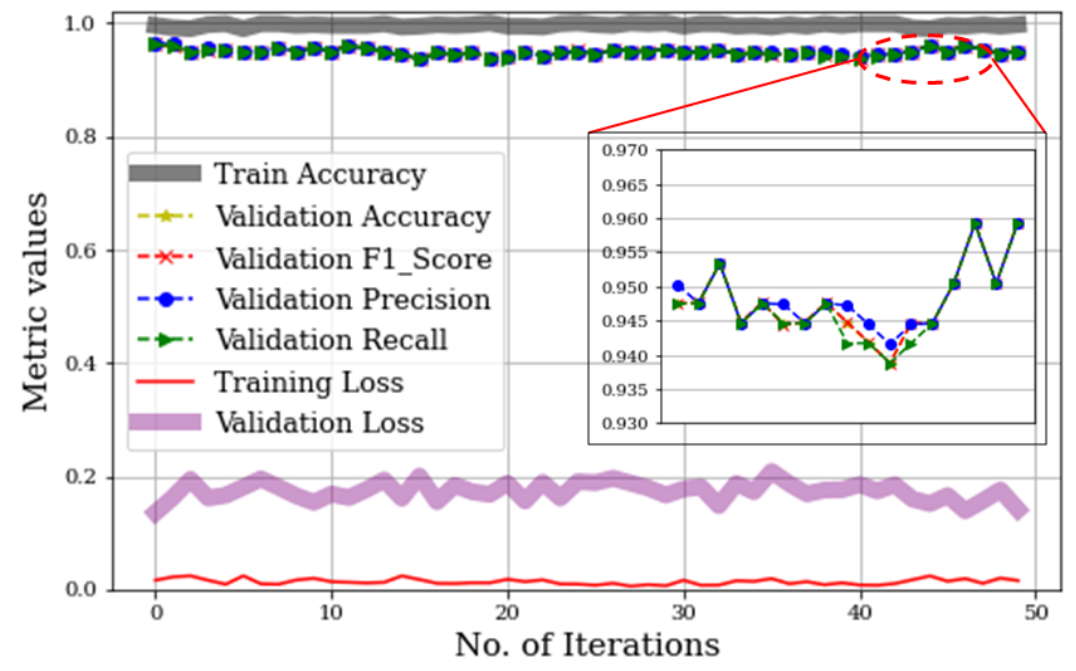

Figure 6. Model training/validation history showing accuracy, loss, precision, recall, and F1-score. 
As observed, the validation accuracy, recall, precision, and F1-scores range between $93.7 \%$ and $96 \%$ throughout the training process with an average validation loss of $20 \%$ (thick purple line). From the zoomed view in Figure 6 above, it can also be observed that the accuracy and losses do not change significantly over the number of iterations until about the 45th iteration where the accuracy increases to a maximum of $96 \%$. The authors believe the marginal difference in accuracy (and loss) levels over the iteration is as a result of quick error convergence of by the model give the high discriminance in the training inputs. Over a 10-fold cross validation, the average accuracy, recall, precision, and F1-scores were recorded as $95.918 \%, 95.921 \%, 95.915 \%$, and $95.921 \%$ respectively.

Although these global metrics are quite intuitive, the need for an in-depth assessment of the developed model prompted the authors to conduct assessments on the high-level features extracted from the Conv layers. First, a correlation-based assessment of the features were conducted and the results presented in Figure 7. Next, explainable paradigms are employed for local and feature-based model assessments.
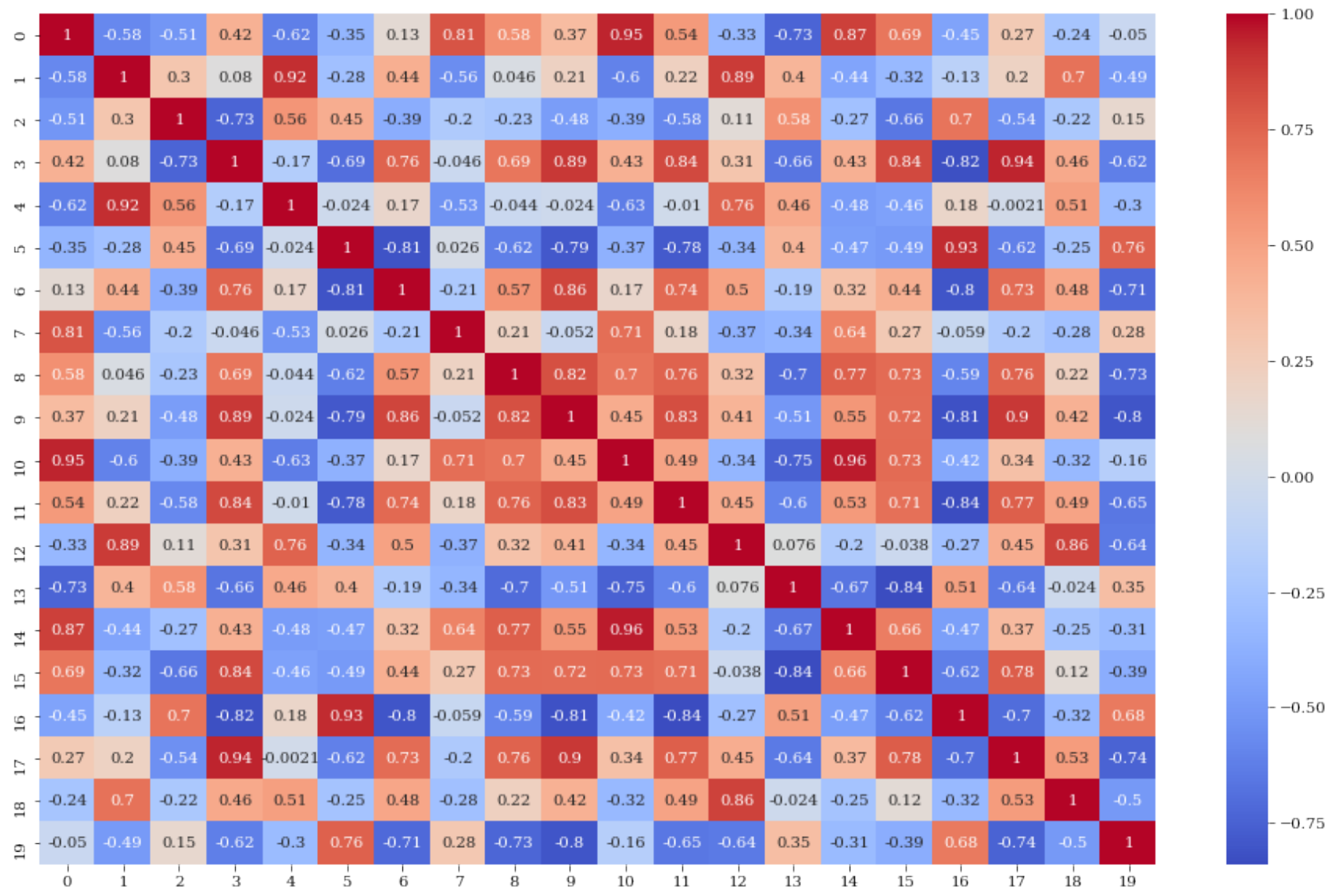

Figure 7. Correlation matrix of the high-level features.

As shown, most of the features have negative correlation values (in blue) accompanied by a few other feature pairs with low positive correlation (in light red). These invariably suggest a high discriminance amongst the features and further goes to validating the efficiency of the Conv layers for discriminative high-level feature extraction.

\subsection{Performance Evaluation}

Model inspection for understanding the rationale behind its decision logic has become important for real-life applications. To evaluate the proposed model's performance, a comparative assessment with other popular high-performing DL models were employed. It is worth noting that these models- gated recurrent units(GRU), long short-term memory (LSTM), and bi-directional LSTM (BI-LSTM) share a similar architecture with the proposed 
$\mathrm{CNN}$ model in the sens e that they have the same number of nodes and layers (besides the convolutional layers). This provides a relatively fair standpoint for which an ideally fair comparison would be made since it would be futile to explore all the possibilities for the algorithms. In this light, a global evaluation was conducted based on the overall test scores and the computational costs associated with each algorithm during the analysis. Figure 8a presents the global test accuracy comparison while Figure $8 \mathrm{~b}$ presents the computational costs reported for each algorithm over a 10- fold cross validation.

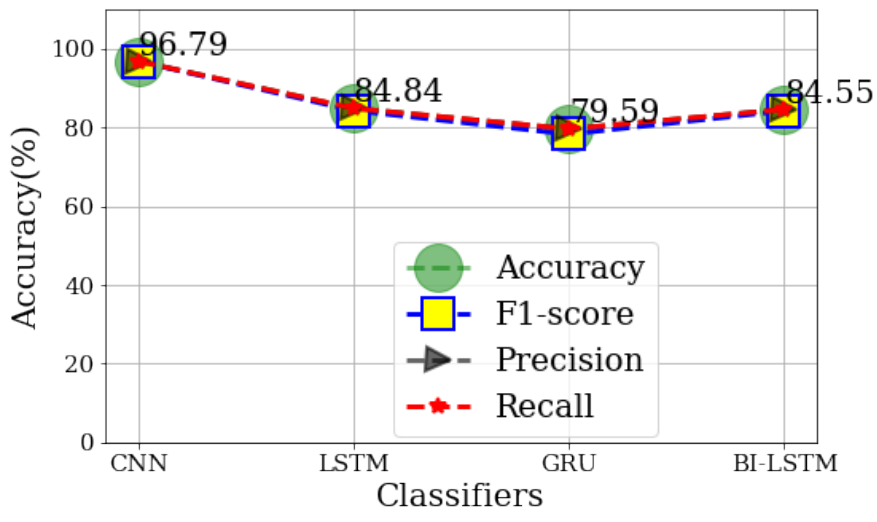

(a)

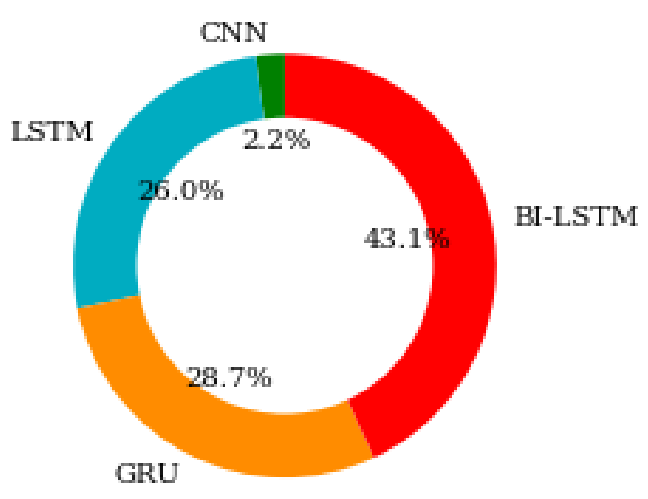

(b)

Figure 8. Global performance comparison of algorithms (a) Overall test performance; (b) Computational costs.

It is observed in Figure 8a that for each of the models, similar test accuracy, F1-score, precision, and recall were returned; however, the clear superior predictive capabilities of the proposed model is observed by $\approx 96.79 \%$ score while the LSTM model ranks second with an accuracy of $\approx 88.84 \%$. On the other hand, it is observed from Figure $8 \mathrm{~b}$ that the stand-alone $\mathrm{CNN}$ models is the most computationally efficient with just $2.2 \%$ (54.4 s) whereas the computational costs for the other models are quite significant with the BI-LSTM's greed for computational resources constituting $\approx 43.1 \%(1060.8 \mathrm{~s})$. All analyses were conducted in the Python-based DL library-Keras with Tensorflow back-end to provide compatibility with GPU environments on a PC with specification-AMD Ryzen 7 (manufactured in Taiwan), 2700 Eight-core $3.20 \mathrm{GHz}$ processor and 16 GB RAM.

In addition to the global prediction performances by the models, a more class-specific evaluation was conducted using confusion matrices and are presented in Figure 9.

As shown in Figure 9a, out of the $14.29 \%$ (100\%/7 classes) perfect prediction for each class, $13.24 \%, 13.99 \%$, and $13.41 \%$ true positives were returned by the model for EM- $1.3 \%$, EM-1.5\%, and EM-2.0\% respectively while perfect predictions are observed for EM- $0 \%$, EM-5.0\%, EM-10.0\% and EM-20.0\% respectively. On the other hand, it is observed from the predictions by the other models as shown in Figure $9 \mathrm{~b}-\mathrm{d}$ that the only the TPs for EM- $0 \%$ and EM- $20.0 \%$ were perfect $(14.29 \%$ ) while for the other emulsion compositions, poor predictions are observed ; especially for EM-1.3\% whereby the FPs are higher than the TPs.

Contrary to the shallow explanation provided by the already-explored metrics and confusion matrix, the LIME algorithm provides a more in-depth avenue for ensuring the faithfulness/trustworthiness of the model is well assessed. Since it provides local interpretability for feature-based (and global) predictions, and being a more reliable stateof-the-art prediction evaluation algorithm for most classifiers and regressors, its judgement is better preferred. In addition to the sample prediction trustworthiness, it returns the feature importance and their contributions to the predictions. Figure 10 show the feature importance and trustworthiness of the sample predictions on different (randomly selected) test samples for each of the emulsion compositions. 


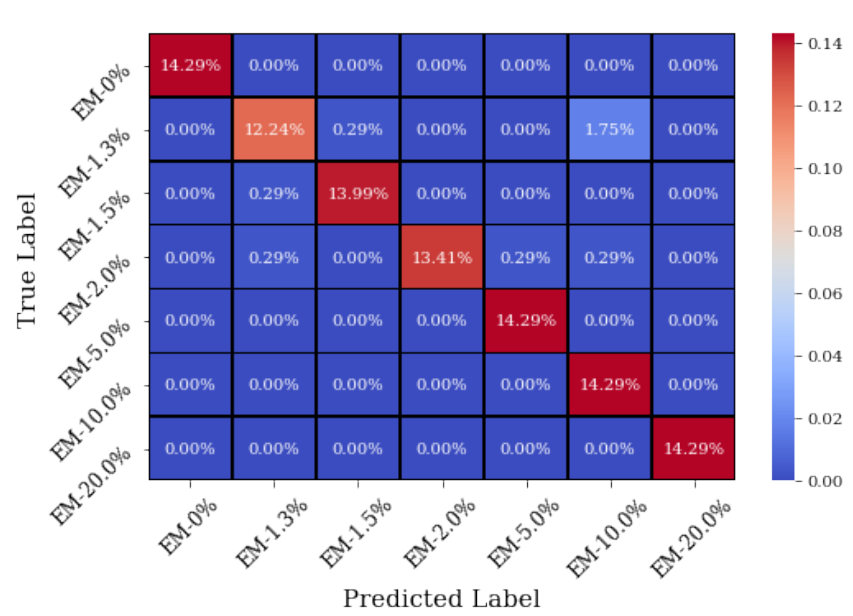

(a)

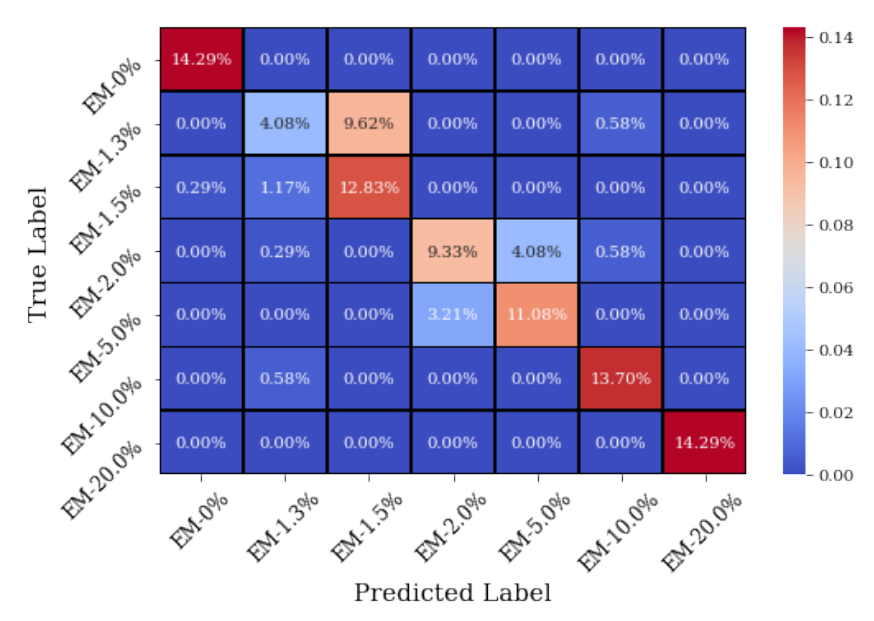

(c)

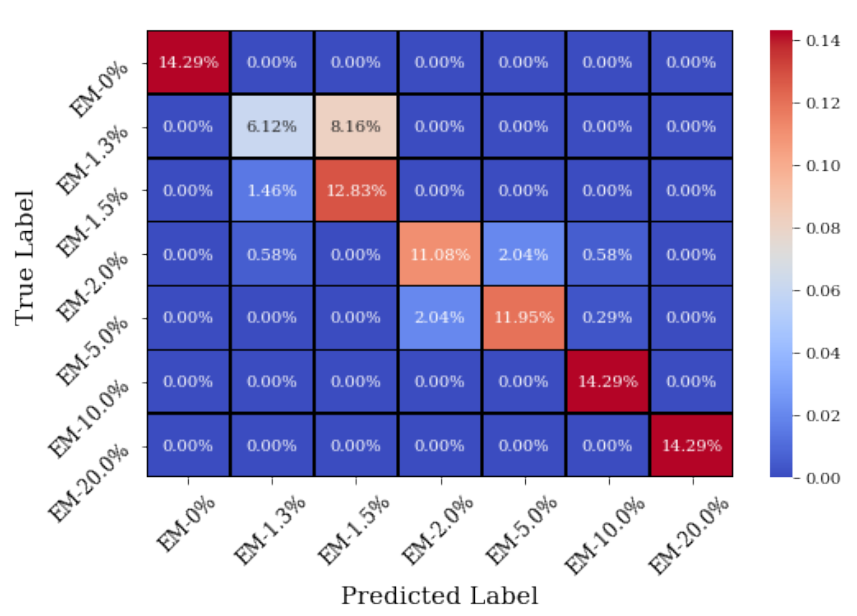

(b)

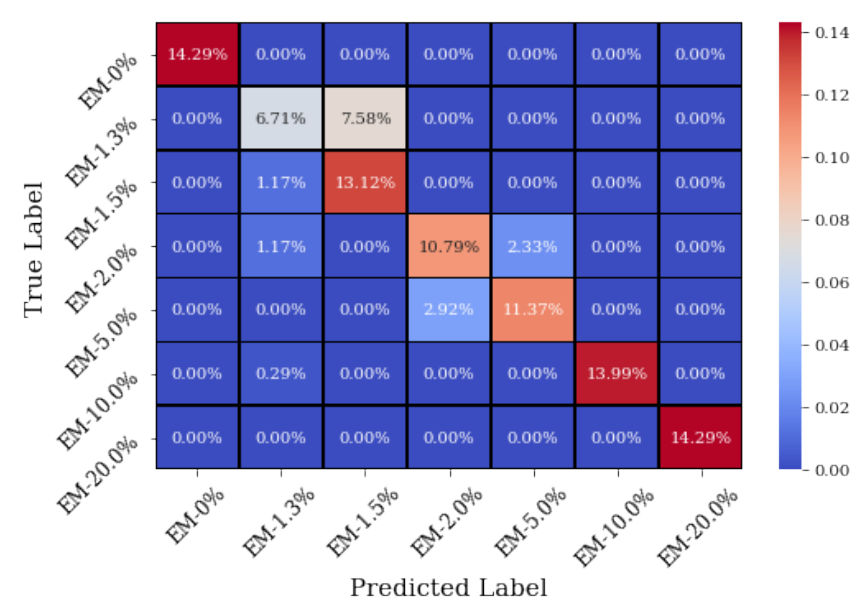

(d)

Figure 9. Confusion matrices on test samples from (a) CNN (b) LSTM (c) GRU and (d) BI-LSTM.

As shown, the algorithm does not only provide the prediction probabilities of the model on the respective samples (for each class), it also reveals the feature importance/ contributions to the predictions. This way, human interpretations of the model's trustworthiness and feature assessments can be conducted. For instance, a closer look at Figure 10a shows that for the single row explanation provided therein for EM-1.3\%, the model is $87 \%$ confident that the input corresponds to EM- $1.3 \%$ while it is only $11 \%$ confident that it corresponds to EM-1.5\%. Invariably, a clear explanation for why the model predicted the inputs to be EM-1.3\% is provided visually while also revealing the features (and their contributions) for making the prediction. It also reveals that for EM-0\% EM-1.3\%, EM-5.0\%, and EM-20.0\% (see Figure 10b, Figure 10e, and Figure 10g, respectively) most of the important (the highest contributing) features are found in the higher engine speeds (1700-2000 RPM) whereas for EM-1.5\% and EM-10.0\% (see Figure 10c and Figure 10f, respectively), the most important features are found within lower engine speeds (1200 RPM). 


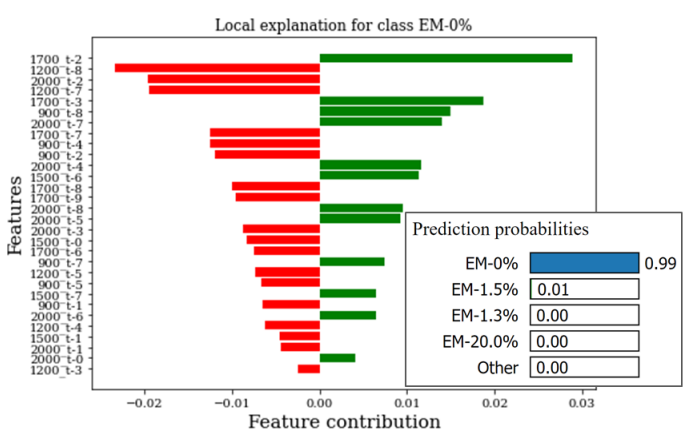

(a)

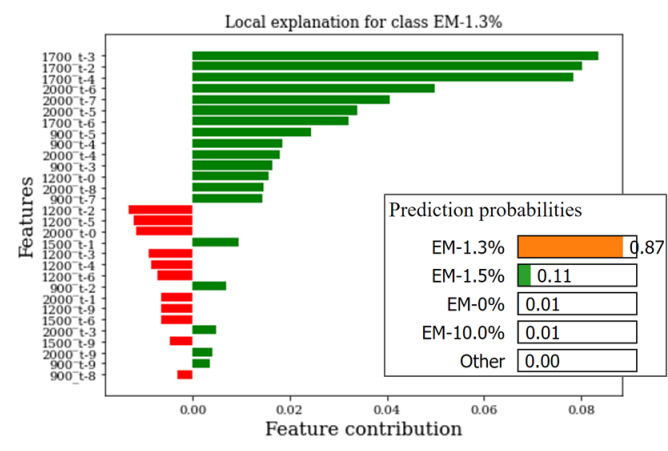

(b)

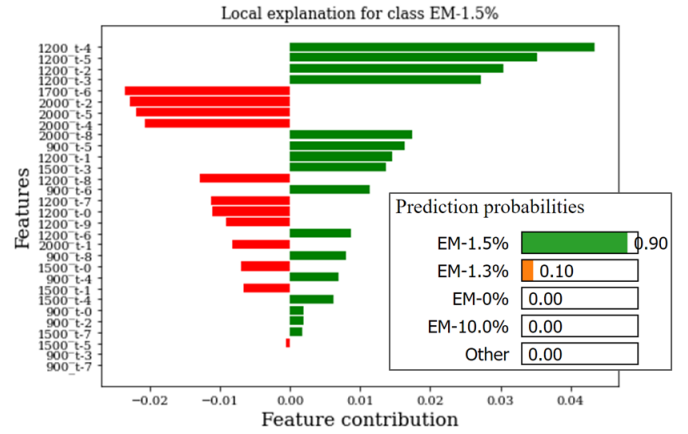

(c)

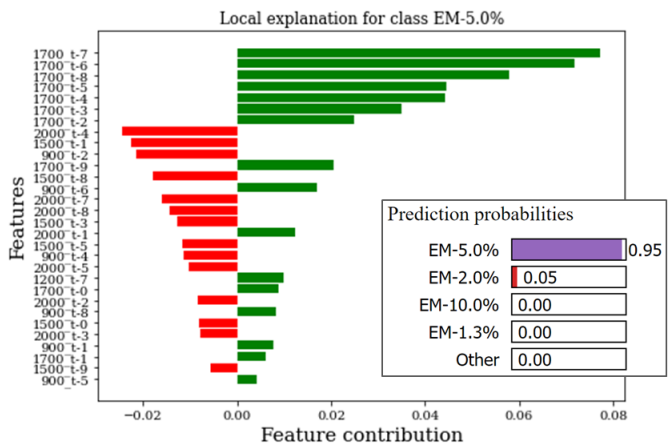

(e)

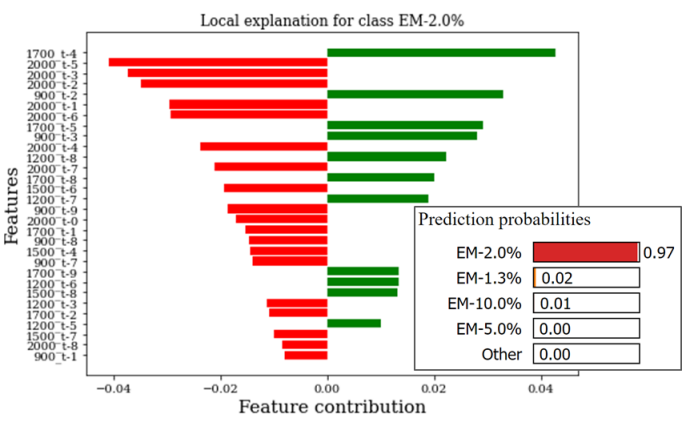

(d)

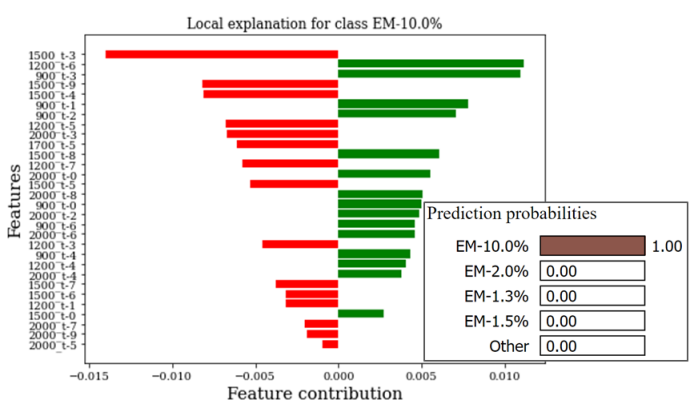

(f)

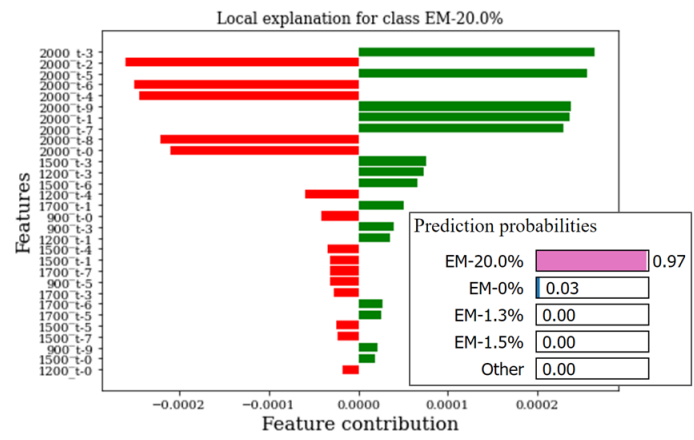

$(\mathbf{g})$

Figure 10. LIME-based explanations, faithfulness, and feature importance/contributions for the proposed model predictions on samples of the different emulsion compositions. (a) EM-0\%, (b) EM-1.3\%, (c) EM-1.5\%, (d) EM-2.0\%, (e) EM-5.0\%, (f) EM-10.0\%, and (g) EM-20.0\%. 


\section{Related Works, Discussions and Open Issues}

A closer look into water emulsified diesel fuels reveals that diesel chemical property is improved by emulsification and this can be attributed to the phenomena- micro explosions. At high temperatures, emulsion droplets evaporate faster which invariably triggers puffing and micro-explosions to produce smaller droplets that improve miscibility between both fluids. In turn, the high production of the pollution-causing compounds are reduced $[9,27]$.

A study by Greeves et al. [28] suggests that water emulsified fuels resulted in a 70\%, $50 \%$, and $50 \%$ reduction of $\mathrm{NO}, \mathrm{CO}$, and smoke, respectively, accompanied with small improvements in specific fuel consumption. Furthermore, their study provided a leeway for other researches on investigating the effect and advantages of several water/diesel mixture ratios $[7,9,29]$. Some of these studies include Azimi et al.'s investigation on the effect of water emulsified diesel fuels on exhaust emissions and engine noise using a water-cooled, four-stroke, four cylinder-engine (direct injection type). Their investigation showed that a $2 \%$ water-in-diesel emulsion reduced overall engine noise [7]. Another study by Marchitto et al. [29] revealed that using emulsified diesel fuels is associated with a significant reduction and improvement in mixture over-fuelling and fuel efficiency, respectively. Furthermore, the authors in [9] investigated for thermal efficiency and conducted several pollution emission analysis of a light-duty diesel engine generator fueled with water-emulsified diesel. Their study recorded a (18.3 to 45.4$) \%$ NOx reduction and about (1.2 to 19.9$) \%$ thermal efficiency. These studies all hint on the efficiencies and advantages of using water-emulsified diesel fuels; yet, the challenge of knowing the globally acceptable water composition still remains due to global differences in climate, usability, engine configuration, diesel fuel quality, durability of engine components, and a host of many uncertainties. Nonetheless, two contradictory facts still remain-the use of water-emulsified fuels have eco-friendly benefits on one hand and long-term wear/corrosion effects on injection system components on the other hand. This contradiction is still a major concern for its adoption.

Although existing studies reveal encouraging paradigms for the use of water-emulsified diesel fuels for improved fuel efficiency and a more eco-friendly environment, the reliability of such methods remains open for continued discussions. Issues on stability, mixing conditions, temperature, and mixing compositions greatly influence the reliability of wateremulsified diesel fuels; nonetheless, as this study proposes, using a highly reliable condition monitoring framework may help curtail injection system damage by using acceptable/safe water emulsions to save cost, minimize engine thermal stress, and reduce pollution-causing emissions while it lasts. The information provided in this study validates the use of small water-emulsion compositions (less than $5 \%$ by volume), above which, the CR system may operate less efficiently as reflected by the spectral parameters for higher emulsion compositions. Besides, against the cost-efficiency of using water-emulsified fuels at lower engine speeds, the irregular fluctuations in the CR system at higher engine speeds may result in poor engine performance.

\section{Conclusions}

Diesel engine emissions contain high amounts of harmful compounds which contribute nearly $30 \%$ of greenhouse effects and a host many health and environmental problems. The use of water-emulsified diesel fuels provide a possible solution for curbing these effects yet, due to the corrosion on engine components from water inclusion to diesel fuels, the need for accurate condition monitoring remains high. This study presents a hybrid deep learning-based condition monitoring framework which relies of the use of standardized CR pressure differentials as inputs and 1D-CNN classifier for diagnostics.

Using a KIA Sorento 2004 four-cylinder line engine as a case study. CR pressure signals were digitally extracted at various water-in-diesel emulsion compositions at various engine RPMs. Extensive empirical assessments were conducted on the proposed model for: 
- Understanding the spectral dynamics in the CR system as a result of diesel emulsification using FFT, PSD and spectral statistical features over different water-in-diesel compositions and engine speeds.

- Exploring (and validating) the proposed diagnostic model for $\mathrm{CR}$ diagnosis given the CR pressure differentials on a real case study.

- Providing explanations on the proposed model's reliability and trustworthiness. These were achieved using diverse conventional diagnostic evaluation metrics and the LIME algorithm. In addition, the cost implications were assessed form a comparative standpoint between the proposed model and other deep learning models.

On a different note, the results from the case study presented in this study were achieved under passive parameter controls-operating conditions and model parameters. Since the emulsion compositions were pre-designed and the model parameters chosen arbitrarily, future research would emphasize on the automation potentials of the proposed model to avoid human errors. Furthermore, to achieve generality of the proposed model, additional tests on different engine models (and configuration) may be needed to fully ascertain with surety the damage severity of diesel emulsification on diesel engines fuel injection system.

From a generalization point of view, the authors believe that beyond the presented case study, the proposed algorithm can flourish (with minor re-configuration) on different applications ranging from fault detection, fraud detection, anomaly detection, and more interestingly, for separation of the emulsion during operations. This is yet another open research path very fertile for exploration and shall motivate our continued research in the domain.

Author Contributions: conceptualization, U.E.A.; methodology, U.E.A.; software, U.E.A.; formal analysis, U.E.A.; investigation, U.E.A.; resources, U.E.A. and J.-W.H.; data curation, U.E.A.; writingoriginal draft, U.E.A., writing - review and editing, U.E.A. and J.-W.H; visualization, U.E.A.; supervision, J.-W.H.; project administration, J.-W.H.; funding acquisition, J.-W.H. All authors have read and agreed to the published version of the manuscript.

Funding: This research was supported by the MSIT (Ministry of Science and ICT), Korea, under the Grand Information Technology Research Center support program (IITP-2020-2020-0-01612) supervised by the IITP (Institute for Information \& communications Technology Planning \& Evaluation).

Data Availability Statement: The data presented in this study are available on request from the corresponding author. The data are not publicly available due to laboratory regulations.

Acknowledgments: Early preliminary research was supported by Min Seop Kim affiliated with the Department of Mechanical Engineering (Department of Aeronautics, Mechanical and Electronic Convergence Engineering), Kumoh National Institute of Technology.

Conflicts of Interest: The authors declare no conflict of interest.

\section{Appendix A. CR Pressure Signal Characteristics}

This document presents the raw CR pressure characteristics using FFT and PSD for the various water emulsion compositions at different engine speeds. Figures A1-A5 present these results for 900, 1200, 1500, 1700, and 2000 RPMs, respectively.

On the left columns of each figure are the standardized CR pressure signals. On the middle are their corresponding FFT spectra while the PSDs are presented on the far right columns. Across the rows are the time-series signal, FFT, and PSD outputs for the respective water emulsion compositions (represented with unique colors as shown in the figure labels at the extreme right corner). As shown, the dynamics in their respective timeseries characteristics can be visually observed; however, the spectral characteristics help provide a more reliable understanding of the $\mathrm{CR}$ system whereby the various constituent frequencies in the time-series are visually observed in the FFT output. This is further evaluated by the respective PSDs which provide the spectral energy distribution found in the signals. It can be observed that across the different conditions, the signals oscillate 
predominantly at lower frequencies $(0-200 \mathrm{~Hz})$ and this is due to the non-stationarity in the signals.
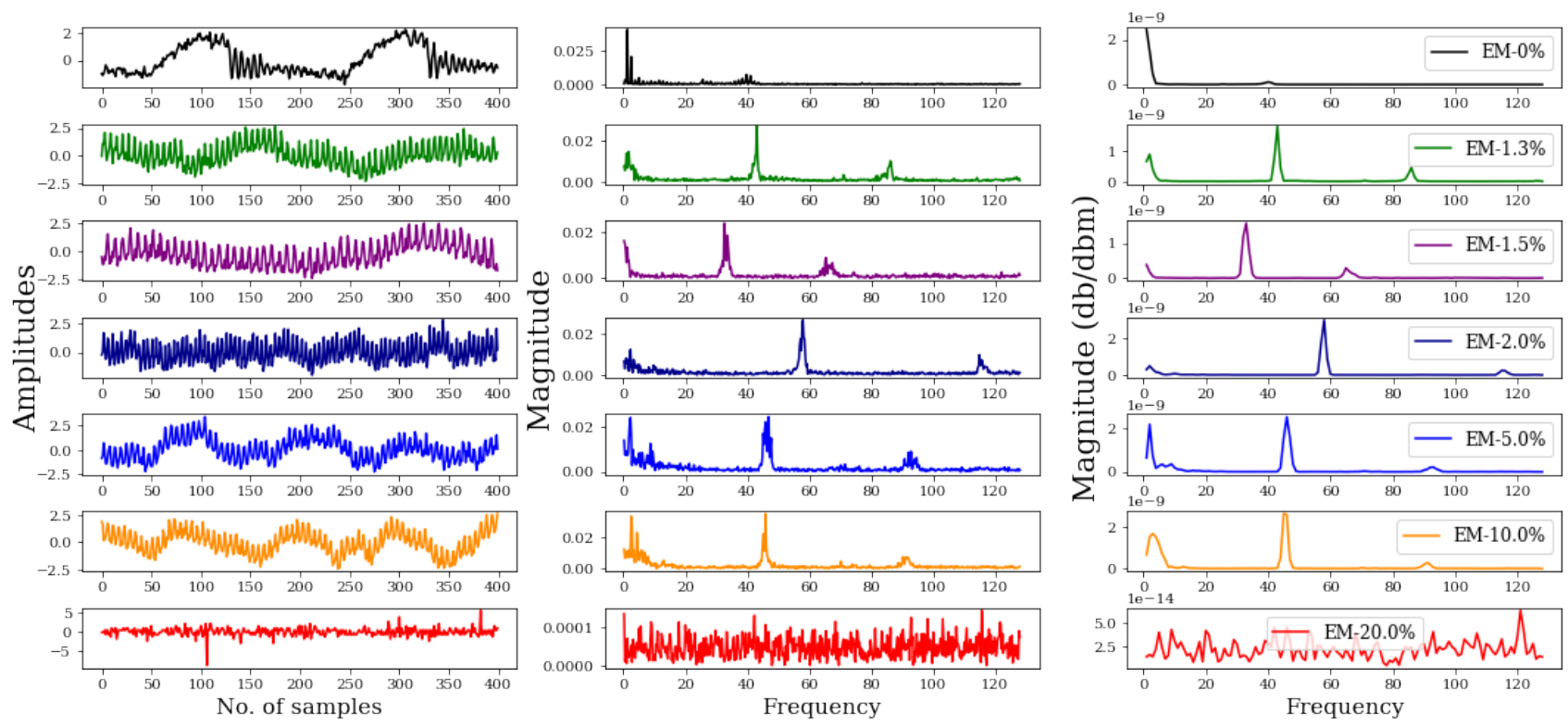

Figure A1. CR signals and their FFT and PSD outputs at 900 RPM for the emulsion compositions.
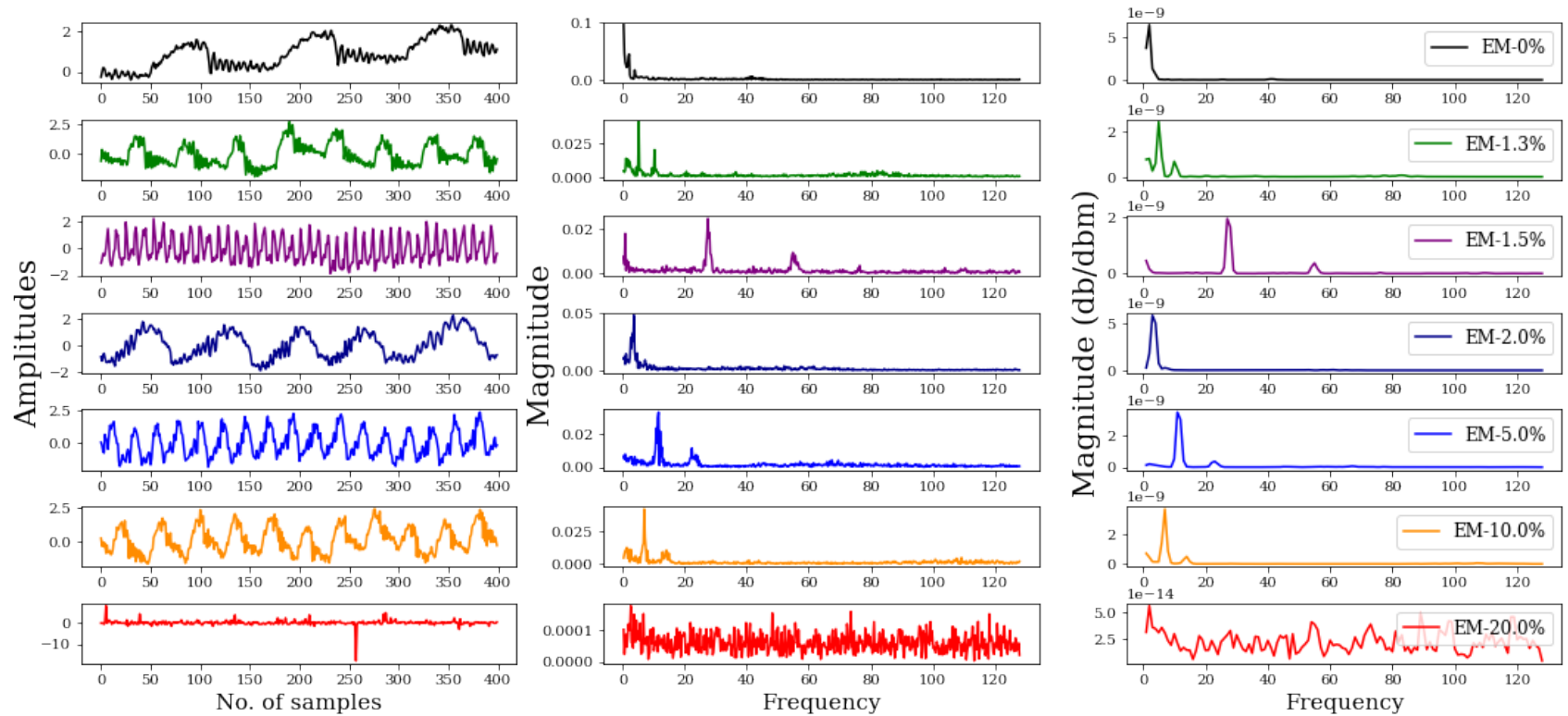

Figure A2. CR signals and their FFT and PSD outputs at 1200 RPM for the emulsion compositions. 

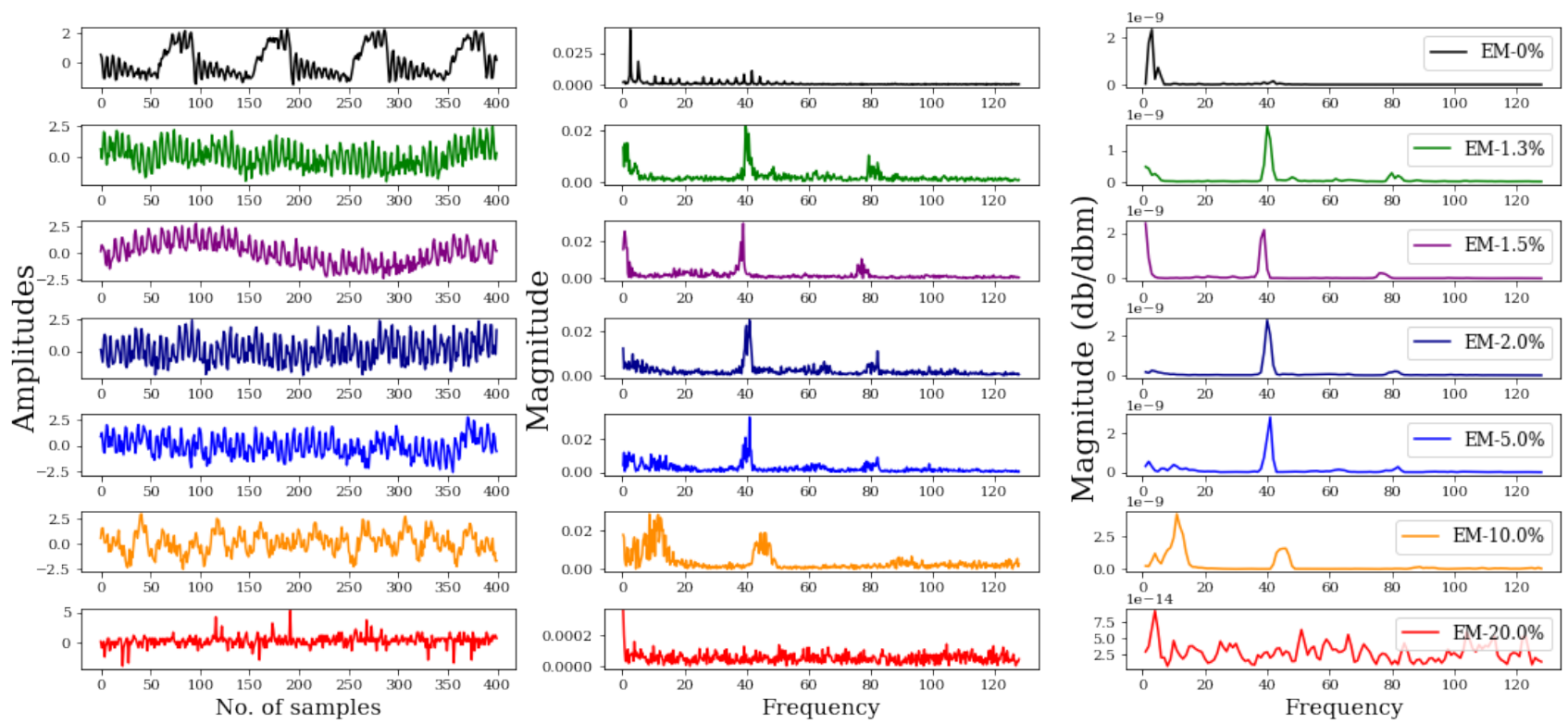

Figure A3. CR signals and their FFT and PSD outputs at 1500 RPM for the emulsion compositions.
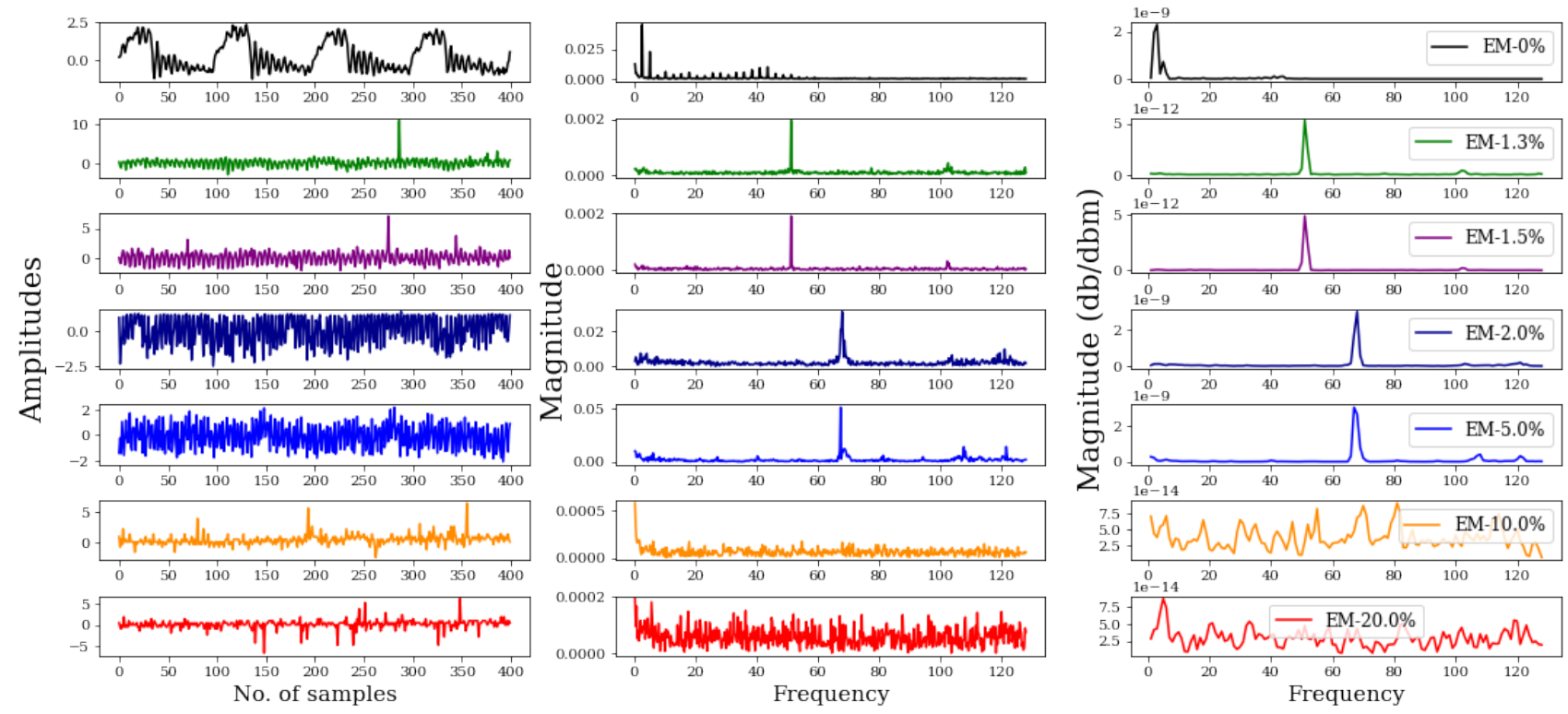

Figure A4. CR signals and their FFT and PSD outputs at 1700 RPM for the emulsion compositions. 

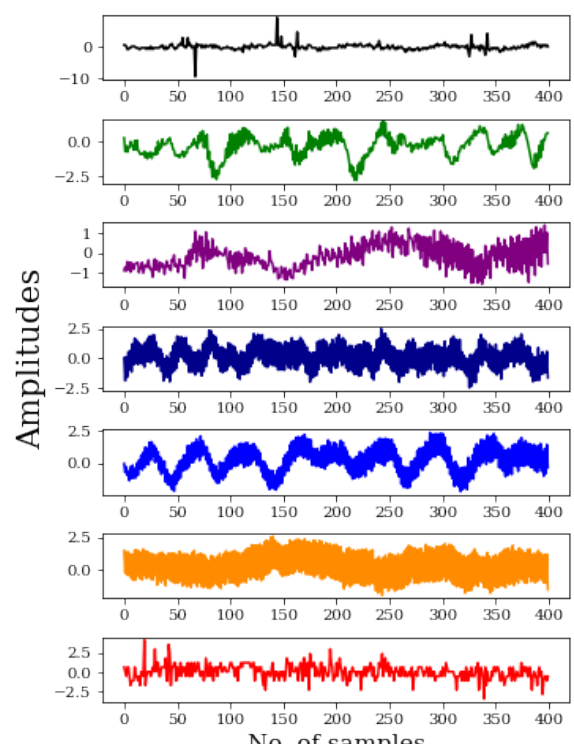
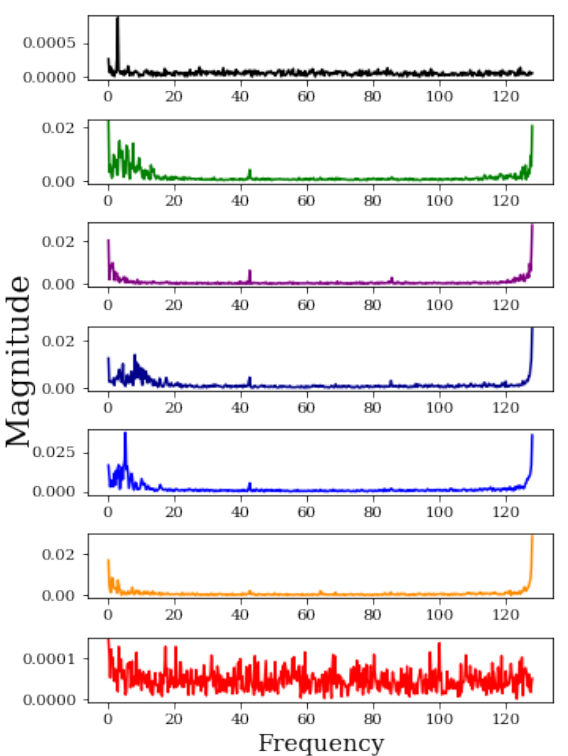

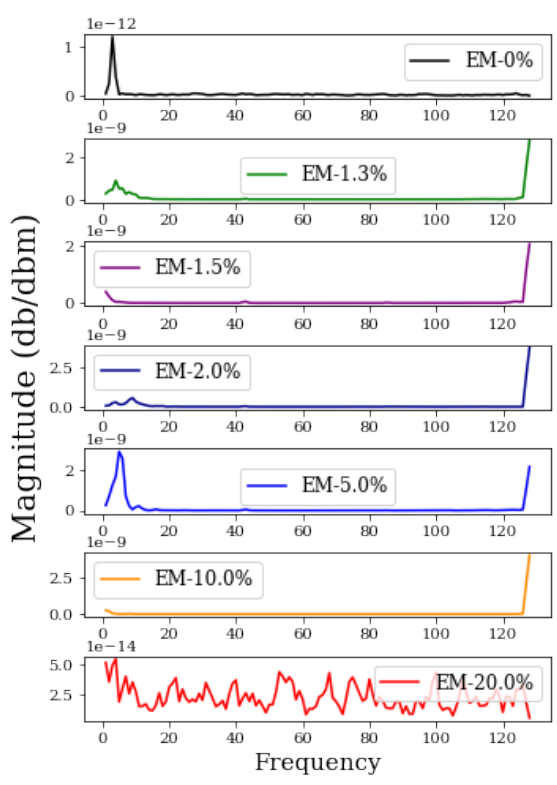

Figure A5. CR signals and their FFT and PSD outputs at 2000 RPM for the emulsion compositions.

\section{Appendix B. CR Pressure Differential Characteristics}

This document presents the $\mathrm{CR}$ pressure differentials and their corresponding spectra (using FFT and PSD) for the various water emulsion compositions at different engine speeds. Figures A6-A10 present these results for 900, 1200, 1500, 1700, and 2000 RPMs, respectively. Similar to the arrangement in the figures Appendix A, the pressure differentials are presented on the left columns of each figure while on the middle and far right columns, are their corresponding FFT and PSD spectra.

As shown the non-stationary behaviour observed in the raw $\mathrm{CR}$ pressure signals are eliminated by extracting differentials. In addition, unique spikes are observed across the different emulsion conditions (and speeds); hence suggesting the discrimnance achieved from computing the pressure differentials. In addition, this inherent discriminance and stationarity better enhances the diagnostic efficiencies of any classifier.
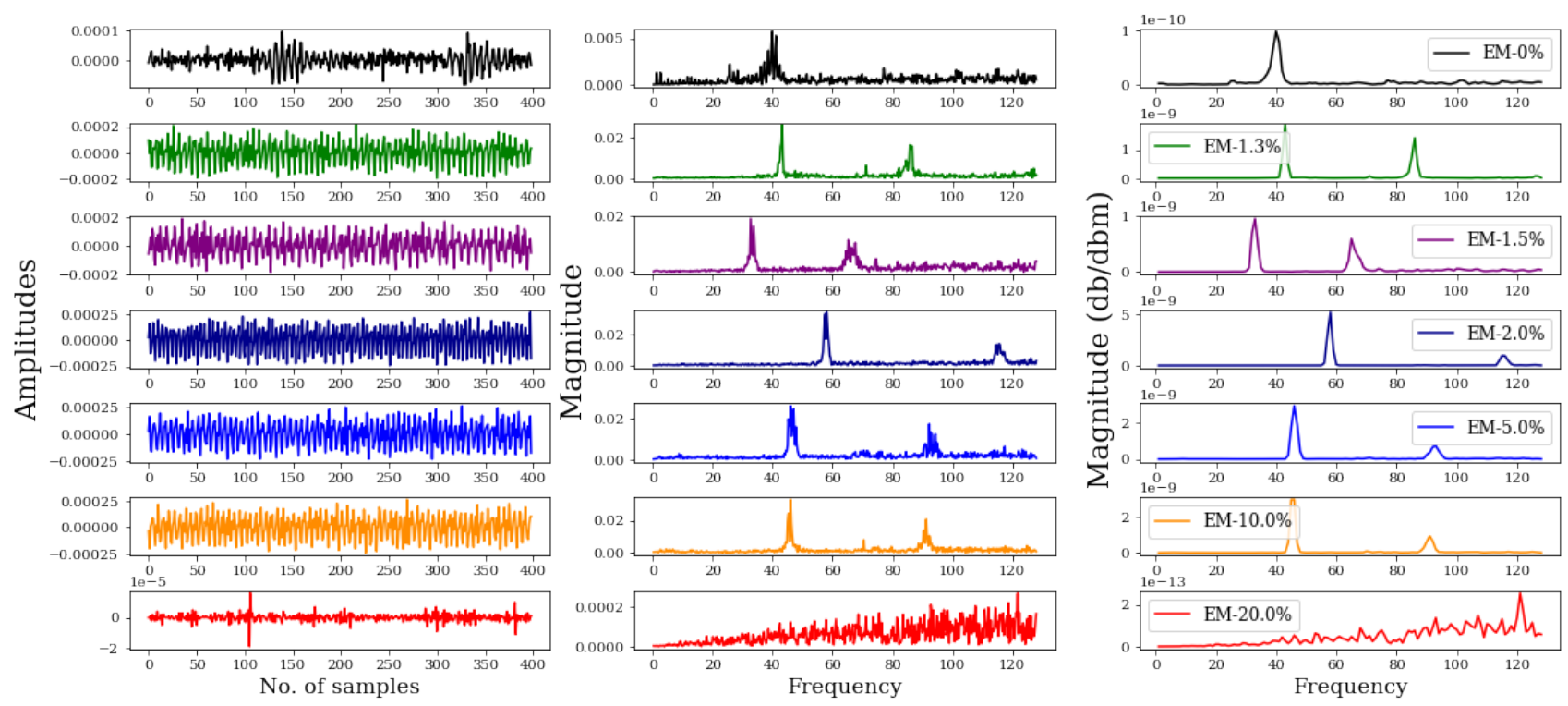

Figure A6. CR differentials and their FFT and PSD outputs at 900 RPM for the emulsion compositions. 

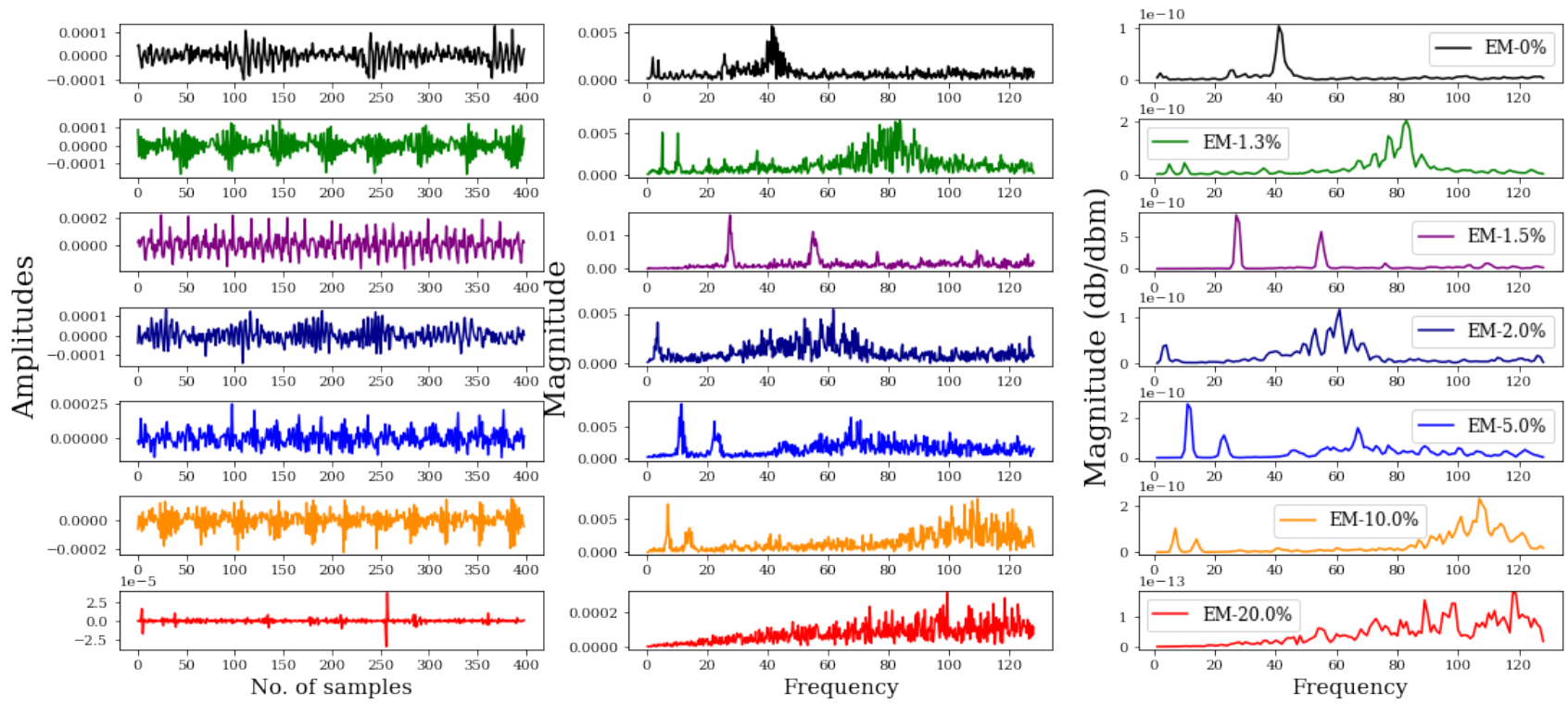

Figure A7. CR differentials and their FFT and PSD outputs at 1200 RPM for the emulsion compositions.
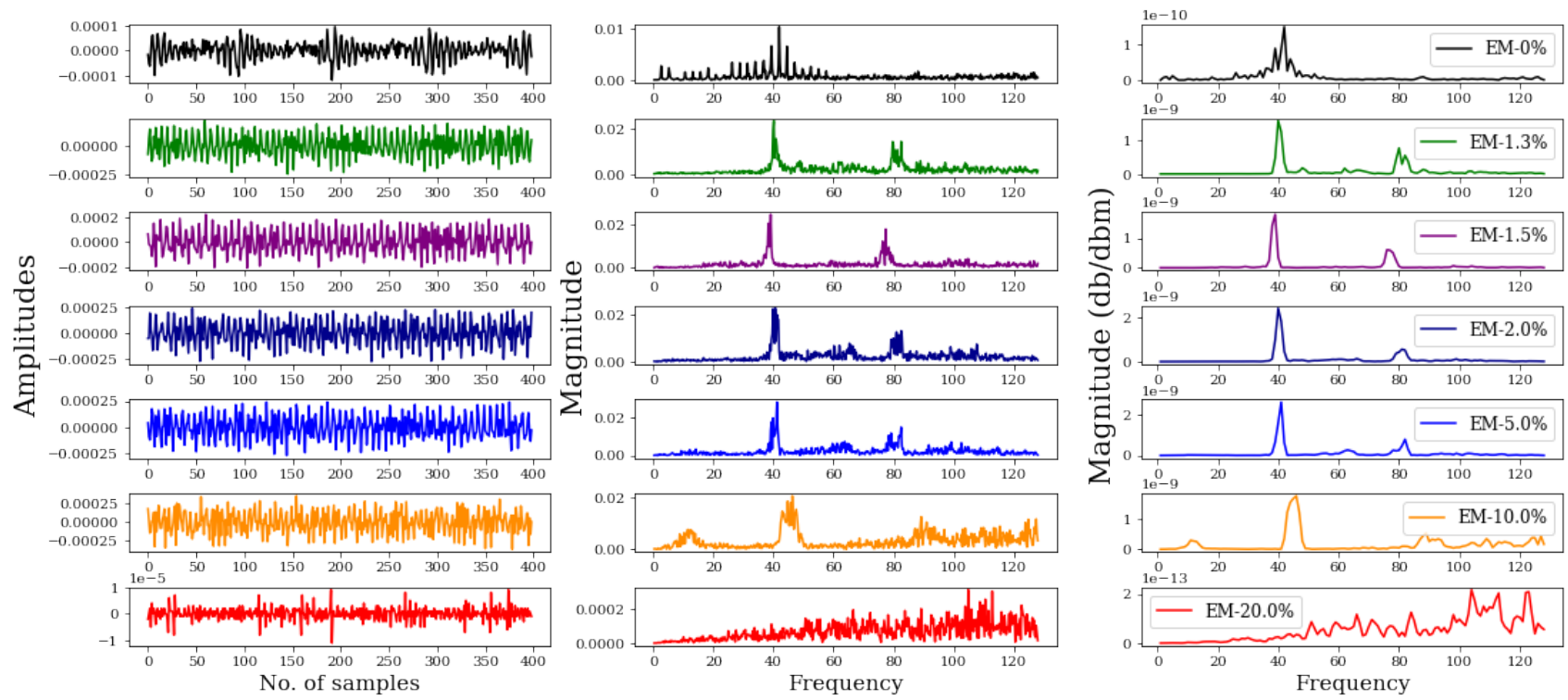

Figure A8. CR differentials and their FFT and PSD outputs at 1500 RPM for the emulsion compositions. 

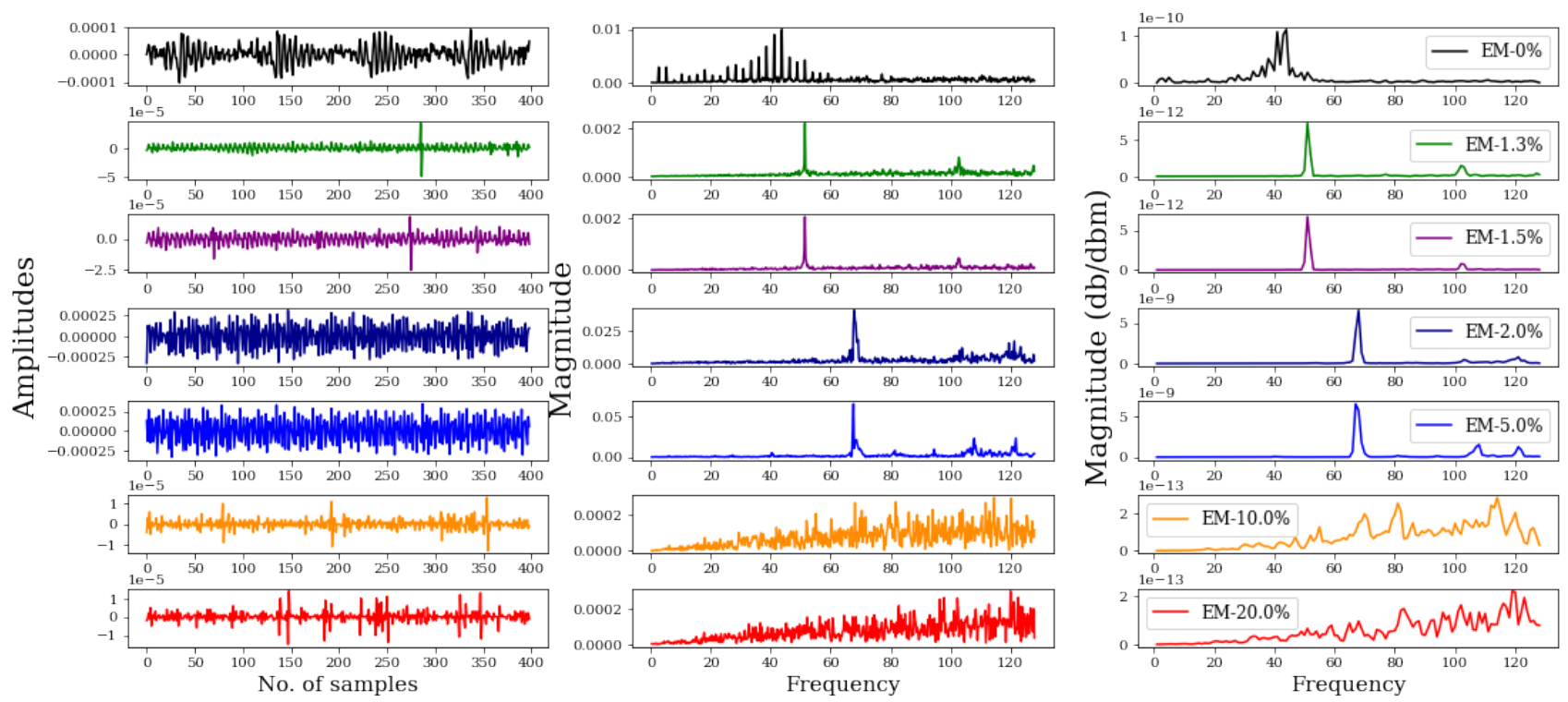

Figure A9. CR differentials and their FFT and PSD outputs at 1700 RPM for the emulsion compositions.
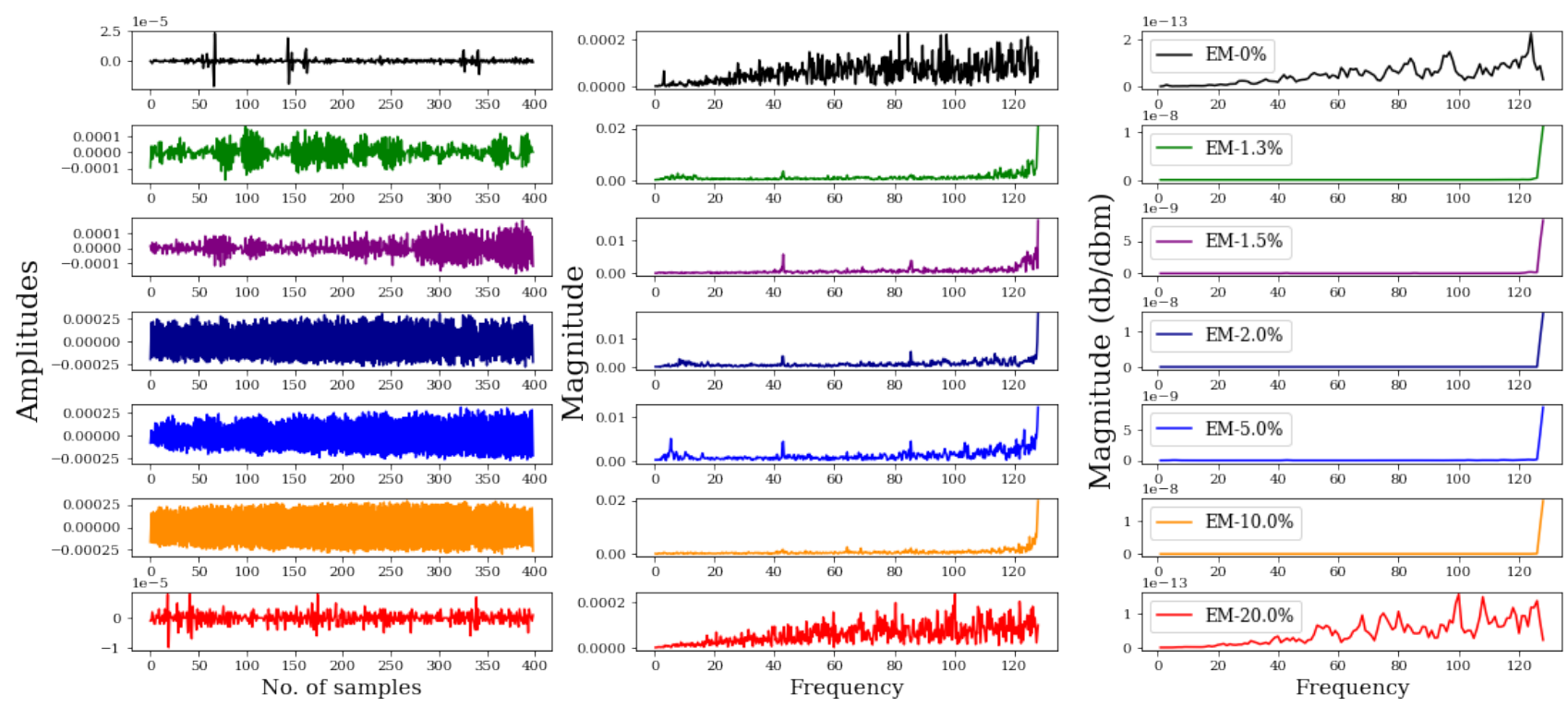

Figure A10. CR differentials and their FFT and PSD outputs at 2000 RPM for the emulsion compositions.

\section{References}

1. Krogerus, T.; Huhtala, K. Diagnostics and Identification of Injection Duration of Common Rail Diesel Injectors. Open Eng. 2018, 8, 1-6. [CrossRef]

2. Krishnamoorthi, M.; Malayalamurthi, R. The influence of charge air temperature and exhaust gas recirculation on the availability analysis, performance and emission behavior of diesel-bael oil-diethyl ether blend operated diesel engine. J. Mech. Sci. Technol. 2018, 32, 1835-1847. [CrossRef]

3. Hossain, F.M.; Nabi, M.N.; Rahman, M.M.; Bari, S.; Van, T.C.; Rahman, S.M.A.; Rainey, T.J.; Bodisco, T.A.; Suara, K.; Ristovski, Z.; et al. Experimental Investigation of Diesel Engine Performance, Combustion and Emissions Using a Novel Series of Dioctyl Phthalate (DOP) Biofuels Derived from Microalgae. Energies 2012, 12, 1964. [CrossRef]

4. Ryu, K.; Oh, Y. A study on the usability of biodiesel fuel derived from rice bran oil as an alternative fuel for IDI diesel engine. KSME Int. J. 2003, 17, 310-317. [CrossRef]

5. Kalghatgi, G.; Johansson, B. Gasoline compression ignition approach to efficient, clean and affordable future engines. Proceedings of the Institution of Mechanical Engineers.Part D J. Automob. Eng. 2018, 232, 118-138. [CrossRef]

6. Song, E.; Ke, Y.; Yao, C.; Dong, Q.; Yang, L. Fault Diagnosis Method for High-Pressure Common Rail Injector Based on IFOA-VMD and Hierarchical Dispersion Entropy. Entropy 2019, 21, 923. [CrossRef] 
7. Azimi, M.; Mirjavadi, S.S.; Davari, E.; Seifi, M.R. The effect of water-diesel emulsion usage on a tractor engine performance and emission. Russ. Agricult. Sci. 2016, 42, 488-492. [CrossRef]

8. Iordache, R.; Petrea, N.; Bujoreanu, C. Wear's issues on high-pressure common rail pumps. In Proceedings of the International Conference on Tribology (ROTRIB' 19), Cluj-Napoca, Romania, 19-21 September 2019. [CrossRef]

9. Syu, J.Y.; Chang, Y.Y.; Tseng, C.H.; Yan, Y.L.; Chang, Y.M.; Chen, C.C.; Lin, W.Y. Effects of water-emulsified fuel on a diesel engine generator's thermal efficiency and exhaust. J. Air Waste Manag. Assoc. 2014, 64, 970-978. [CrossRef]

10. Li, A.; Zheng, Z. Effect of Spark Ignition Timing and Water Injection Temperature on the Knock Combustion of a GDI Engine. Energies 2020, 13, 4931. [CrossRef]

11. Çiçek, K.; Turan, H.H.; Topcu, Y.; Searslan, M.N. Risk-based preventive maintenance planning using Failure Mode and Effect Analysis (FMEA) for marine engine systems. In Proceedings of the 2010 Second International Conference on Engineering System Management and Applications, Sharjah, United Arab Emirates, 30 March-1 April 2010; pp. 1-6.

12. Suresh, V.; Amirthagadeswaran, K.S. The role of water-in-diesel emulsion and its additives on diesel engine performance and emission levels: A retrospective review. Alex. Eng. J. 2016, 55, 2463-2472. [CrossRef]

13. Shyam, P.H.; Joseph, G.; Vijay, V.S. Effect of Introduction of Water into Combustion Chamber of Diesel Engines-A Review. Energy Power 2015, 5, 28-33. [CrossRef]

14. Sundaram, K.M.; Hussain, A.; Sanjeevikumar, P.; Holm-Nielsen, J.B.; Kaliappan,V.K.; Santhoshi, B.K. Deep Learning for Fault Diagnostics in Bearings, Insulators, PV Panels, Power Lines, and Electric Vehicle Applications-The State-of-the-Art Approaches. IEEE Access 2021, 9, 41246-41260. [CrossRef]

15. Akpudo, U.E.; Hur, J.W. An Automated Sensor Fusion Approach for the RUL Prediction of Electromagnetic Pumps. IEEE Access 2021, 9, 38920-38933. [CrossRef]

16. Ribeiro, M.T.; Singh, S.; Guestrin, C. "Why Should I Trust You?": Explaining the Predictions of Any Classifier. arXiv 2016, arXiv:1602.04938.

17. Kim, M.-S.; Akpudo, U.E.; Hur, J.-W. A Study on Water-Induced Damage Severity on Diesel Engine Injection System Using Emulsified Diesel Fuels. Electronics 2021, 10, 2285. [CrossRef]

18. Kim, J.; Kim, J.; Kim, H.; Shim, M.; Choi, E. CNN-Based Network Intrusion Detection against Denial-of-Service Attacks. Electronics 2020, 9, 916. [CrossRef]

19. Hasan, M.N.; Toma, R.N.; Nahid, A.-A.; Islam, M.M.M.; Kim, J.-M. Electricity Theft Detection in Smart Grid Systems: A CNN-LSTM Based Approach. Energies 2019, 12, 3310. [CrossRef]

20. Yuan, C.M.; Sun, X.M.; Zhao, H. Speech Separation Using Convolutional Neural Network and Attention Mechanism. Discret. Dyn. Nat. Soc. 2020, 2020, 2196893. [CrossRef]

21. Chen, C.C.; Liu, Z.; Yang, G.; Wu, C.C.; Ye, Q. An Improved Fault Diagnosis Using 1D-Convolutional Neural Network Model. Electronics 2021, 10, 59. [CrossRef]

22. Wang, Y.; Ma, S.; Shen, X. A Novel Video Face Verification Algorithm Based on TPLBP and the 3D Siamese-CNN. Electronics 2019, 8, 1544. [CrossRef]

23. Akpudo, U.E.; Hur, J.W. Towards bearing failure prognostics: A practical comparison between data-driven methods for industrial applications. J. Mech. Sci. Technol. 2020, 34, 4161-4172. [CrossRef]

24. Tjoa, E.; Guan, C. A Survey on Explainable Artificial Intelligence (XAI): Toward Medical XAI. IEEE Trans. Neural Netw. Learn. Syst. 2020, 1-21. [CrossRef] [PubMed]

25. Lee, N.; Azarian, M.H.; Pecht, M.G. An Explainable Deep Learning-based Prognostic Model for Rotating Machinery. arXiv 2020, arXiv:2004.13608.

26. Gronfors, T.; Sihvonen, T.; Lamsa, V.; Paivinen, N. Spectral Factors and Medical Parameters of EMG Signals. In Proceedings of the 2006 IET 3rd International Conference On Advances in Medical, Signal and Information Processing-MEDSIP 2006, Glasgow, UK, 17-19 July 2006; pp. 1-4. [CrossRef]

27. Madan, M.A.; Thanos, M.; Jun, X.; Ganippa, L. Experimental understanding on the dynamics of micro-explosion and puffing in ternary emulsion droplets. Fuel 2019, 239, 1284-1292. [CrossRef]

28. Greeves, G.; Khan, I.M.; Onion, G. Effects of water introduction on diesel engine combustion and emissions. Symp. (Int.) Combust. 1977, 16, 321-336. [CrossRef]

29. Marchitto, L.; Tornatore, C.; Costagliola, M.A.; Lacobacci, A. Effect of Water Injection on Fuel Efficiency and Gaseous and PN Emissions in a Downsized Turbocharged SI Engine. J. Energy Eng. 2018, 144, 04018044. [CrossRef] 